\title{
Políticas para reducir el problema (no percibido) de las personas ubicadas en Áreas Vulnerables
}

\section{Policies to reduce the problem (not perceived) of people located in Vulnerable Areas}

\author{
José Robles Laynes \\ Universidad Nacional Mayor de San Marcos, Facultad de Ciencias Sociales. Lima, Perú \\ Email: robles_josel@hotmail.com
}

\section{Resumen}

Este trabajo parte de la interrogante: ¿Cuál sería la razón por la cual nuestros compatriotas optan viven en áreas vulnerables, exponiendo su vida y la de sus familiares? Esta pregunta es relevante porque afecta a millones de compatriotas (36\% de la población, según el Ministerio del Ambiente) y es aplicable a cualquier parte del Perú.

Para responder a esta pregunta se propone un modelo explicativo, señalándose variables de análisis, y sus posibles relaciones, para luego realizar simulaciones. Es necesario considerar que el objetivo de las simulaciones no es brindar resultados exactos, sino describir posibles comportamientos futuros, sobre la base de las acciones que tomemos en el presente.

El modelo planteado considera un horizonte de 50 años, e indica que sólo si se trata el problema en forma intensiva, el porcentaje de personas en áreas vulnerables podría reducirse luego de una década. Asimismo, se concluye que si no se toman acciones, las personas ubicadas en áreas vulnerables representarían el $51 \%$ de la población en el periodo analizado. En ese sentido, las políticas que más aportarían a reducir el porcentaje de personas en áreas vulnerables son: la reubicación, la promoción de la educación y la mejora en la eficacia del gasto público.

Palabras clave: Demografía; factores gubernamentales; grupos sociales; simulación de sistemas.

\begin{abstract}
This research work starts with the question: What would be the reason why our compatriots choose to live in vulnerable areas, exposing their own life and that of their relatives? This question is relevant because it affects millions of compatriots (36\% of the population, according to the Ministry of the Environment) and is applicable to any part of Peru.

To answer the question an explanatory model is proposed, indicating variables of analysis, and their possible relationships, to make simulations, from the model. It is necessary to consider that the objective of the simulations is not to provide exact results, but to describe possible future behaviors, considering the actions that we take in the present.

The proposed model considers a horizon of 50 years, and indicates that only if the problem is treated intensively the percentage of people in vulnerable areas could be reduced after a decade. Likewise, it is concluded that if actions are not taken, people located in vulnerable areas would represent $51 \%$ of the population in the period analyzed. In that sense, the policies that would contribute to reduce the percentage of people in vulnerable areas are: relocation, education and the improvement in the effectiveness of public spending.
\end{abstract}

Keywords: Demography; Governmental factors; Social Groups; Systems simulation.

Correspondencia:

Dirección: Universidad Nacional Mayor de San Marcos, Facultad de Ciencias Sociales. Calle Germán Amézaga N³75, Ciudad Lima 1.

Recibido 17/10/2018 - aceptado 06/03/2019

Citar como:

Robles, J. (2019) Políticas para reducir el problema (no percibido) de las personas ubicadas en Áreas Vulnerables. Revista Peruana de Computación y Sistemas, 2(1):37-56. http://dx.doi.org/10.15381/rpcs.v2i1.16434

(c) Los autores. Este artículo es publicado por la Revista Peruana de Computación y Sistemas de la Facultad de Ingeniería de Sistemas e Informáticade la Universidad Nacional Mayor de San Marcos. Este es un artículo de acceso abierto, distribuido bajo los términos de la licencia Creative Commons Atribucion - No Comercia_Compartir Igual 4.0 Internacional. (http://creativecommons.org/licenses/by-nc-sa/4.0/) que permite el uso no comercial, distribución y reproducción en cualquier medio, siempre que la obra original sea debidamente citada. 


\section{Introducción}

$\mathrm{Si}$, en un día soleado paseamos por cualquiera de las zonas periféricas (y no tan periféricas) de Lima o provincias, si miramos con atención vamos a encontrar (quizá sin sorprendernos) que el paisaje también está conformado por viviendas, comercios, colegios, y hasta instituciones públicas que han optado por colocarse cerca (o dentro) de un área peligrosa, exponiéndose a diferentes tipos de riesgo, ya sea porque se ubican en áreas sujetas a derrumbes o deslizamientos, o porque se colocan cerca de instalaciones que conllevan riesgos de electrocución o explosiones.

Algunos ejemplos de los hechos mencionados son expuestos en las Figuras: 1, 2, 3 y 4.
Los hechos ilustrados hacen que nos preguntemos: ¿Cuál sería la razón por la cual nuestros compatriotas optan por vivir en áreas vulnerables (como al pie de un acantilado, en el cauce de una quebrada o cerca de una instalación peligrosa), exponiendo su propia vida y la de sus familiares?

En las próximas líneas se tratará de explicar por qué se debe atender este problema de manera prioritaria y se tratará de moldear una respuesta a la interrogante planteada proponiendo un diagrama causal explicativo de la situación de las personas en áreas vulnerables.

Luego de ello, se simulará cómo irían las cosas si no se toma ninguna acción adicional a las que se viene tomando a la fecha, para posteriormente formular algu-

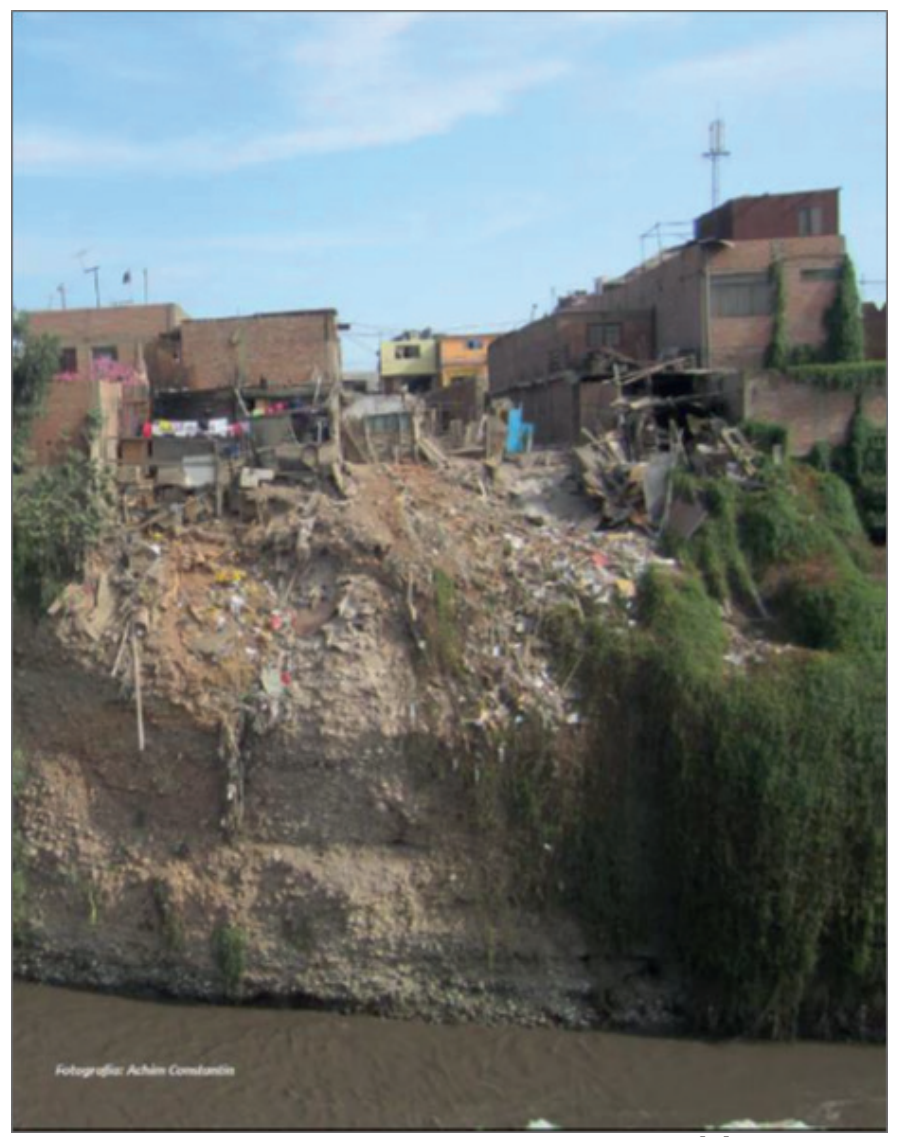

Figura 1. Vivienda al pie de un acantilado [1]

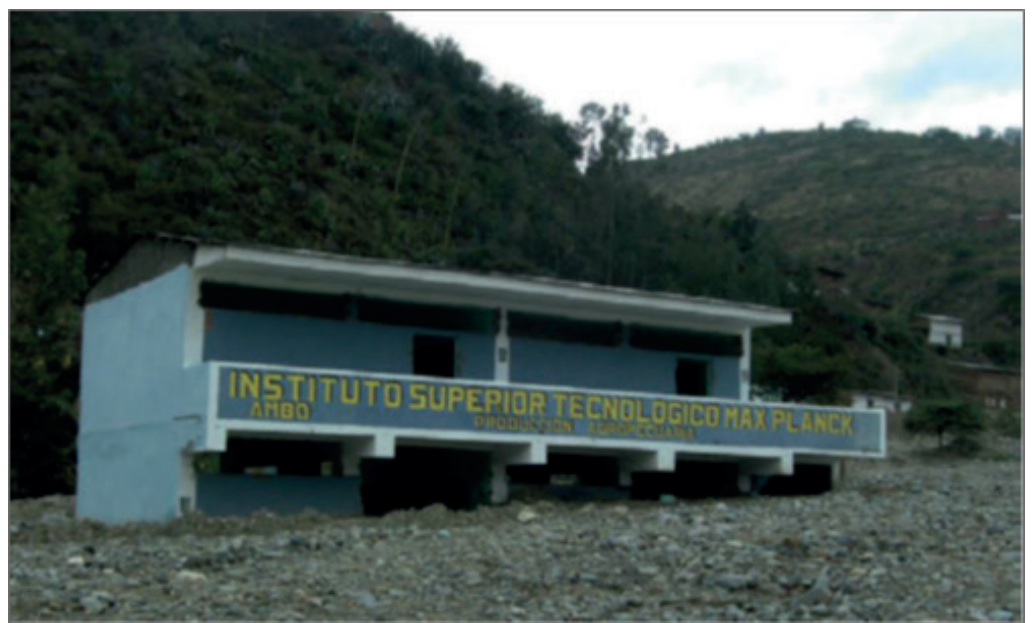

Figura 2. Institución educativa en el curso de una quebrada [2] 


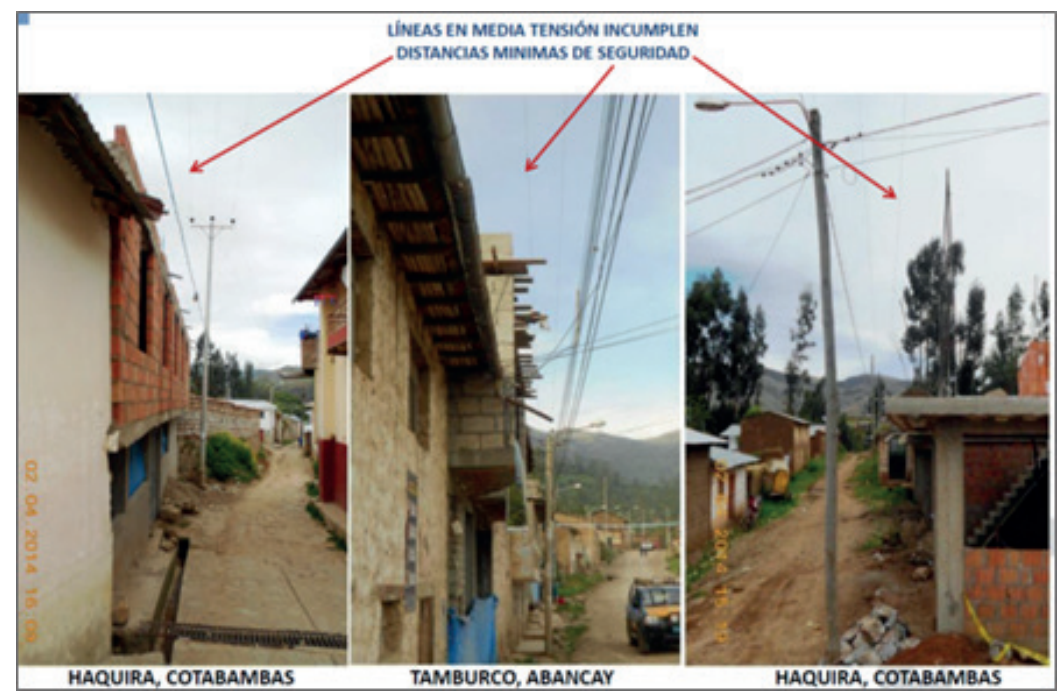

Figura 3. Viviendas expuestas a descargas eléctricas [3]

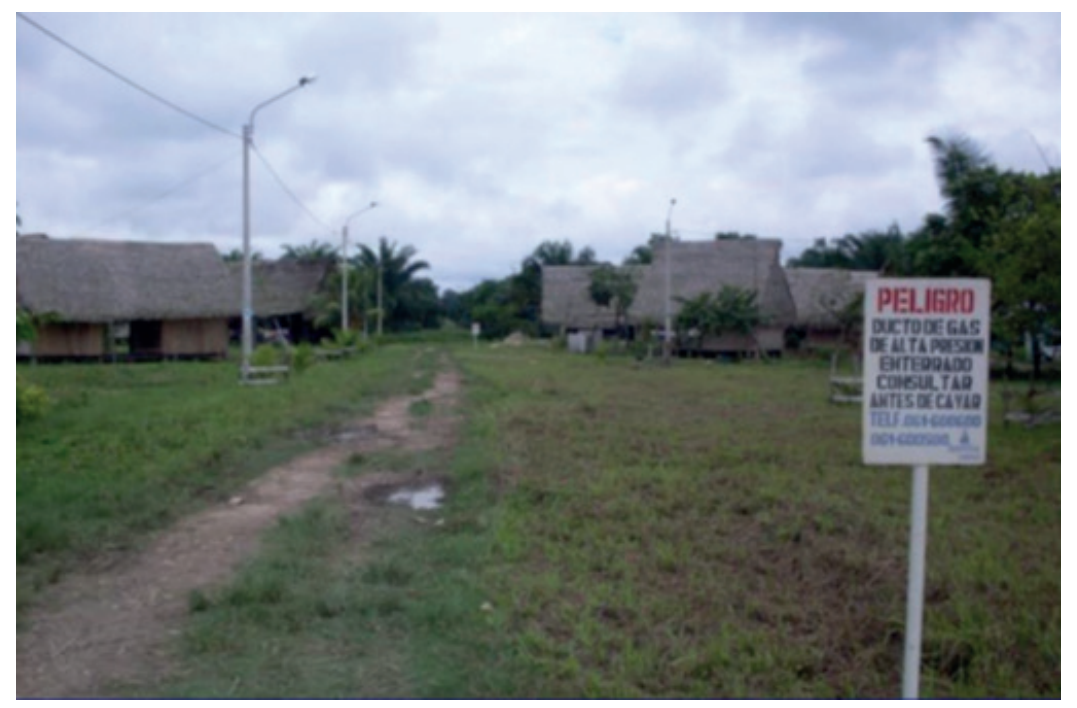

Figura 4. Viviendas y postes de alambrado aledaños al Derecho de Vía de un ducto de gas natural Ducto de gas natural operado por la empresa Aguaytia energy (Archivo Osinergmin).

nas propuestas con la finalidad de reducir el número de personas ubicadas en áreas vulnerables.

\section{Revisión de literatura: ¿Cuántas personas se ubican en áreas vulnerables?}

Uno de los precedentes de este trabajo es el informe "The Limits to growth" [4], el cual presenta los resultados de simular el crecimiento de la población considerando un suministro finito de recursos. A pesar de haber sido actualizado el 2004, y la metodología y predicciones de este informe siguen siendo vigentes. Una de las conclusiones de este informe señala que si no se modifican las tendencias históricas del crecimiento económico, los límites de dicho crecimiento se harán evidentes en la segunda parte del siglo XXI, proyectándose un continuo crecimiento poblacional antes de producirse una repentina e incontrolable reducción de la población y de los recursos naturales.

Asimismo, este artículo considera algunos de los temas abordados en la tesis "Población en Áreas Vulnerables y Política de Gestión de Riesgos: El caso del transporte de gas natural por ductos", cuya hipótesis plantea que el nivel de riesgo para las personas ubicadas en las áreas aledañas a un gasoducto aumenta en el tiempo [5].

\subsection{La población vulnerable}

Para los fines de este trabajo es necesario estimar cuál es la tendencia del número de personas ubicadas en áreas vulnerables para un mediano y largo plazo, por esta razón, se considera un periodo de análisis de cincuenta años, para lo cual se expondrá: el crecimiento del número de personas vulnerables, el número de personas ubicadas en áreas vulnerables, y la tendencia del número de personas ubicadas en áreas vulnerables.

El Plan Nacional de Gestión del Riesgo de Desastres 2014-2021aprobado por Decreto Supremo No 034-2014-PCM, aprobado por la Presidencia del Consejo de Ministros (PCM) identifica y cuantifica a la población vulnerable a nivel nacional, para lo cual utiliza los siguientes criterios: a) Exposición de la población y 
sus medios de vida a los peligros originados por fenómenos naturales y los inducidos por la acción humana; b) Localización de las provincias que presentan alto y muy alto riesgo; c) Población que está en pobreza y extrema pobreza; y d) Evolución de la población afectada y damnificada por emergencias y desastres [6].

El número de personas en condición vulnerable senalado por la PCM es alarmante, sus proyecciones indican que la población vulnerable al año 2021 sería de 21,09 millones de personas, según lo mostrado en la Figura 5.

Los datos proporcionados por la PCM señalan que la tasa anual de crecimiento de la población vulnerable es de $1.55 \%$ (más acelerada que la tasa de crecimiento poblacional de $1.44 \%$ ), por lo cual se espera que en el tiempo el porcentaje de la población vulnerable sea cada vez mayor respecto a la población total.

Nótese que, según lo proyectado, el porcentaje de la población vulnerable al año 2016 resultó del 59\%, y, al año 2021, sería del 64\% del total.

\subsection{Población ubicada en áreas vulnerables}

En la sección anterior se mostró la tendencia de la población vulnerable en Perú, la cual va creciendo a un ritmo más acelerado que el crecimiento poblacional.
Ahora nos toca ver qué porcentaje de la población está ubicado en áreas de vulnerabilidad considerable.

Para estimar el número de personas en áreas vulnerables, consideraremos a la población expuesta a deslizamientos e inundaciones, tomando como referencia los datos indicados en el "Mapa de susceptibilidad física de zonas propensas a inundaciones y deslizamientos en la costa y sierra frente a la ocurrencia de eventos hidrometeorológicos externos" publicado por el Ministerio del Ambiente (MINAM).

Asimismo, de los datos expuestos en la Tabla 1, se puede apreciar que más de 15 mil centros (ya sean educativos o de salud) estarían afectados a deslizamientos e inundaciones, según lo expuesto en la Figura 6.

Los datos de la Tabla 1 también nos permite apreciar que más de 4 millones de viviendas y más de 14 millones de personas son susceptibles de ser afectadas por deslizamientos e inundaciones, según lo expuesto en la Figura 7.

\subsection{Población en áreas de vulnerabilidad alta o muy alta}

Líneas atrás se señaló que algo menos de la mitad de la población está ubicada en áreas vulnerables, lo cual se debe, en parte, a nuestra geografía. En ese sen-

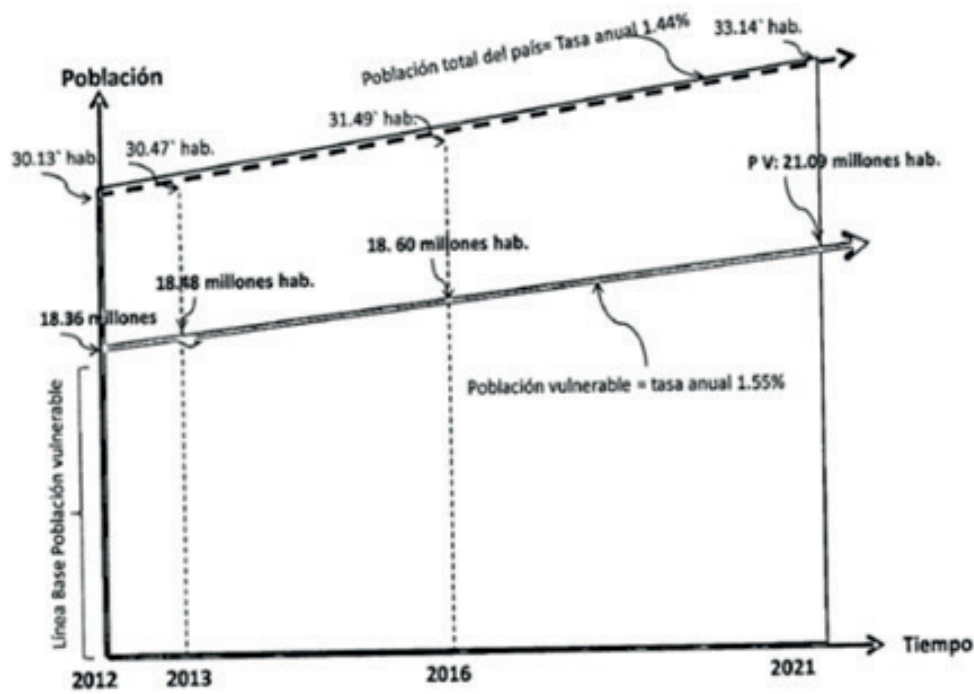

Figura 5. Proyección de la Población Total y Vulnerable al 2021 [6]

Tabla 1. Susceptibilidad a deslizamientos e inundaciones - 2014

\begin{tabular}{|c|c|c|c|c|}
\hline & $\begin{array}{l}\text { Afectación por } \\
\text { Deslizamientos } \\
\text { (i) }\end{array}$ & $\begin{array}{l}\text { Afectación por } \\
\text { Inundaciones } \\
\text { (ii) }\end{array}$ & $\begin{array}{l}\text { Ambos } \\
\text { (iii) }\end{array}$ & $\begin{array}{c}\text { Total afectados } \\
\text { (por deslizamientos y/o inundaciones) } \\
\text { (i) }+ \text { (ii) }\end{array}$ \\
\hline Centros Poblados & 70,966 & 4,701 & 1,216 & 75,667 \\
\hline Viviendas & $2,711,227$ & $1,761,095$ & 252,237 & $4,472,322$ \\
\hline Población en área vulnerable & $8,376,458$ & $6,935,079$ & 935,693 & $15,311,537$ \\
\hline Porcentaje de la Población Total (*) & $27.2 \%$ & $22.5 \%$ & $3.0 \%$ & $49.7 \%$ \\
\hline Centros Educativos & 8,360 & 2,959 & 79 & 11,319 \\
\hline Centros de Salud & 3,241 & 1,157 & 38 & 4,398 \\
\hline
\end{tabular}

$\left({ }^{*}\right)$ Se ha considerado la población en el Perú para el año 2014: 30,814,175 (Fuente: INEI https://proyectos.inei.gob.pe/web/poblacion/) Fuente: [7] / Elaboración propia 


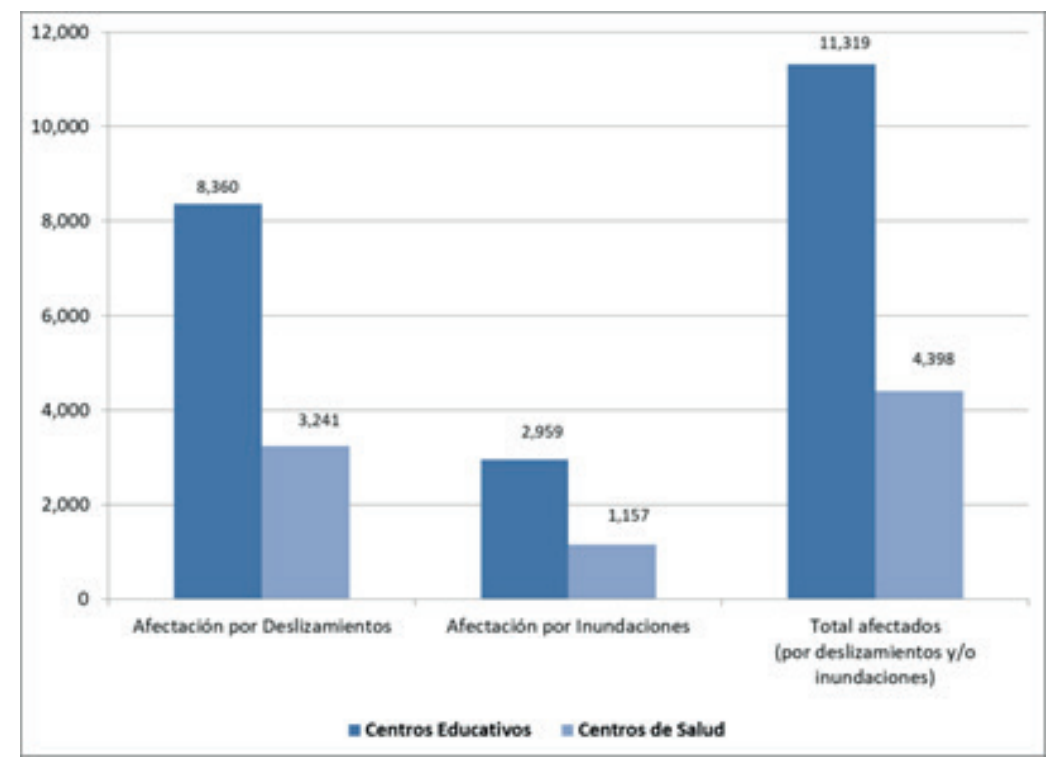

Figura 6. Deslizamientos o inundaciones a centros educativos y de salud Fuente: [7] / Elaboración propia.

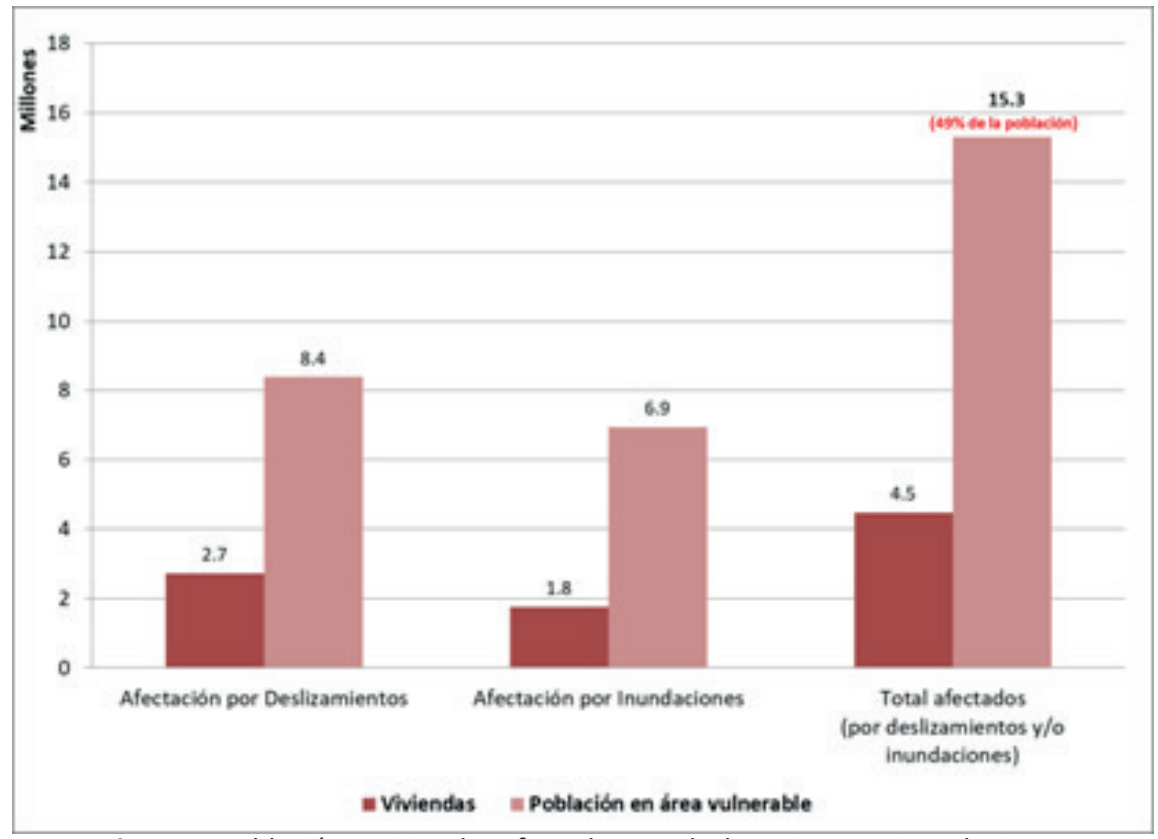

Figura 7. Población y Viviendas afectadas por deslizamientos e inundaciones

Fuente: [7] / Elaboración propia.

tido, el MINAM ha indicado que "el $46 \%$ del territorio nacional se encuentra en condiciones de vulnerabilidad alta a muy alta" [8].

Lo señalado en el párrafo anterior no es un problema menor, sin embargo, es necesario llamar la atención que en estas zonas de alta vulnerabilidad se encuentra el $36 \%$ de la población nacional que "ocupa y hace uso de este espacio territorial" [8].

En ese sentido, si la población que ocupa zonas expuestas a eventos extremos representa el $36 \%$ de la población, el número de personas ubicadas en Áreas Vulnerables en el año 2016 sería de 11’335,905 (11 mi- llones 335 mil 905), valor que resulta al considerar una población de 31’488,625 [6].

De acuerdo a lo indicado en la Figura 5, se considera que la tasa de crecimiento del número de personas en áreas de vulnerabilidad alta o muy alta sería igual a la tasa anual de crecimiento de la población vulnerable: $1.55 \%$.

\section{3. ¿Por qué hay personas en áreas vulnerables?}

Antes de desarrollar este punto, es necesario indicar que la intención de este artículo no es dar una respuesta exacta o completa, sino describir la situación problemática desde un punto de vista particular, que esperamos aporte a la discusión del problema que afecta a nuestros compatriotas ubicados en áreas vulnerables. 


\subsection{Diagrama causal explicativo}

Para tratar de responder al por qué hay personas en áreas vulnerables se desarrolló un diagrama causal que expone un modelo mental explicativo de esta situación.

Antes de pasar a la explicación de las variables, es pertinente recordar al lector los diferentes tipos de relación causal entre una variable de causa (variable-causa) y una variable de efecto (variable-efecto), según lo expuesto en la Tabla 2.

Luego de exponer la definición de los tipos de relaciones en el diagrama causal, corresponde definir las variables expuestas en la figura 8 , según lo expuesto en la Tabla 3:

\subsection{Supuestos del modelo propuesto}

Los supuestos del modelo mostrado en la Figura 8 son los siguientes:
- La “inversión en salud” aumentará (disminuirá) si:

- Se incrementa (reduce) la "eficiencia del gasto en salud”.

- Se incrementa (reduce) la "eficacia del gasto en salud”.

- A mayor (menor) sea la "inversión en salud", menor (mayor) será la "vulnerabilidad a la pobreza”.

- A mayor (menor) sea la "vulnerabilidad a la pobreza”, mayor (menor) será el "número de personas en áreas vulnerables".

- La “inversión en educación” aumentará (disminuirá) si: - Se incrementa (reduce) la "eficiencia del gasto en educación".

- Se incrementa (reduce) la "eficacia del gasto en educación".

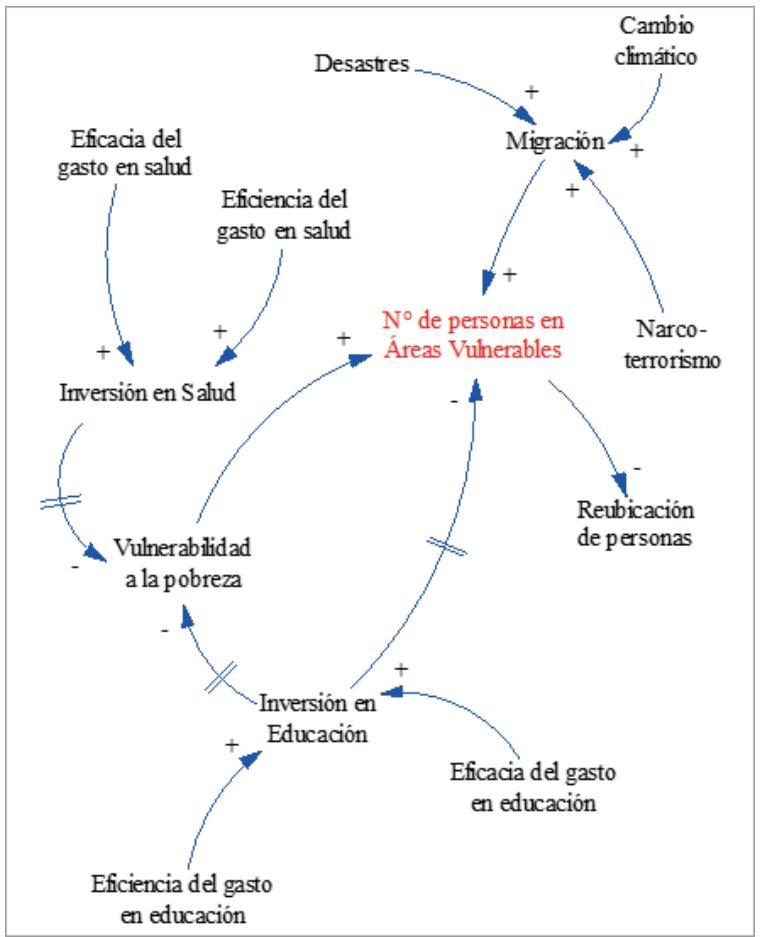

Figura 8. Diagrama causal de las variables que impactarían el número de personas ubicadas en áreas vulnerables [5] Elaboración propia.

Tabla 2. Tipos de relaciones en un diagrama causal

$\begin{array}{ll}\text { Tipo } & \begin{array}{l}\text { Definición } \\ \text { Relación directa o positiva }\end{array} \\ \text { Lariable-efecto también se incrementa; o si, al disminuir la variable-causa, la variable-efecto también se reduce. } \\ \text { Este tipo de relaciones se representa a través de una flecha con un signo positivo (+). } \\ \text { Lelación inversa o negativa } \\ \text { variable-efecto se reduce; o si, al disminuir la variable-causa, la variable-efecto se incrementa. } \\ \text { Este tipo de relaciones se representa a través de una flecha con un signo negativo (-). }\end{array}$

Fuente: [9], [10] 
Tabla 3. Variables relacionadas al número de personas en áreas vulnerables, en el Diagrama Causal

\begin{tabular}{|c|c|}
\hline Variable & Definición \\
\hline Cambio climático & $\begin{array}{l}\text { Fenómeno producido por la variación del clima del planeta generada por la acción del ser humano, el cual } \\
\text { puede producir inundaciones o cambios que provoquen el desplazamiento de personas (migración). }\end{array}$ \\
\hline Desastres & Desastres de origen natural o antrópico que pueden provocar la migración de personas. \\
\hline Eficacia del gasto en educación & $\begin{array}{l}\text { Qué tan bien se ejecutan los gastos en el sector educación, en la medida en que responden a una planifica- } \\
\text { ción previa. }\end{array}$ \\
\hline Eficacia del gasto en salud & $\begin{array}{l}\text { Qué tan bien se ejecutan los gastos en el sector salud, en la medida en que responden a una planificación } \\
\text { previa. }\end{array}$ \\
\hline Eficiencia del gasto en educación & Qué porcentaje de lo previsto para atender al sector educación ha sido efectivamente ejecutado. \\
\hline Eficiencia del gasto en salud & Qué porcentaje de lo previsto para atender al sector salud ha sido efectivamente ejecutado. \\
\hline Inversión en educación. & Porcentaje del PBI del Estado destinado al sector educación. \\
\hline Inversión en salud & Porcentaje del PBI del Estado destinado al sector salud. \\
\hline Migración & $\begin{array}{l}\text { Desplazamiento de personas impulsada por factores como desastres, narco-terrorismo o efectos del cambio } \\
\text { climático. }\end{array}$ \\
\hline Narco-terrorismo & $\begin{array}{l}\text { Situación de terrorismo y/o narcotráfico que puede producir afectaciones a los ductos de gas natural por } \\
\text { vandalismo. }\end{array}$ \\
\hline $\mathrm{N}^{\circ}$ de personas en áreas vulnerables & Número de personas ubicadas en áreas con un alto riesgo de ocurrencia de desastres naturales. \\
\hline Reubicación de personas & $\begin{array}{l}\text { Personas que son reubicadas de las áreas vulnerables debido a políticas o gestiones de las instituciones } \\
\text { estatales. }\end{array}$ \\
\hline Vulnerabilidad a la pobreza & Pérdida de bienestar, respecto a expectativa de vida, analfabetismo y salud. \\
\hline
\end{tabular}

Fuente: [5]

- A mayor (menor) sea la "inversión en educación", se reducirá (aumentará) la magnitud de la "vulnerabilidad a la pobreza”.

- A más (menos) "inversión en educación" haya, se reducirá (aumentará) en mayor medida el "número de personas en áreas vulnerables".

- La "migración” aumentará (disminuirá) si:

- Se incrementa (reducen) la magnitud de la variable "desastres".

- Se incrementa (disminuye) la magnitud de los efectos del "cambio climático".

- Se incrementa (reduce) la magnitud de la variable "narco-terrorismo".

- A mayor (menor) sea la magnitud de la "migración", mayor (menor) será el "número de personas en áreas vulnerables".

- A mayor sea la "reubicación de personas" menor será el "número de personas en áreas vulnerables".

Los supuestos planteados parten de criterios lógicos expuestos en el diagrama causal. A partir de estos supuestos se construirá un modelo de simulación, con la finalidad de evaluar el comportamiento de las variables del modelo en el tiempo.

Se debe tener presente que un diagrama causal no es más que una representación simplificada (un modelo mental) realizada por un observador desde una perspectiva determinada. Por esta razón, puede haber tantos Diagramas Causales como observadores existan. En tal sentido, el diagrama causal expuesto en la figura 8 es una propuesta que trata de explicar el hecho de que haya personas ubicadas en áreas vulnerables.

\section{4. ¿Cuál sería el panorama si se dejan las cosas tal como hasta ahora?}

Para tener una perspectiva del panorama futuro, en caso de que no se atienda el problema de las personas en áreas vulnerables, se expondrá el diagrama causal de tal manera que permita la simulación (Diagrama de Forrester), los resultados de esta simulación son expuestos en las próximas líneas.

Cabe señalar que la simulación presenta, entre otras, las siguientes ventajas: Permite adquirir una experiencia rápida a bajo costo y sin riesgos, identifica "cuellos de botella" en un sistema complejo, permite realizar un estudio sistemático de alternativas, permite plantear estrategias frente a un problema determinado, no tiene límite en cuanto a complejidad, todo sistema, por complejo que sea, puede ser modelizado [11].

\subsection{Transformación del diagrama causal en un Diagrama de Forrester}

En esta etapa se transformará el diagrama causal expuesto en la sección 3 en un Diagrama de Forrester, o de flujos y niveles, con la finalidad de incluir las ecuaciones del modelo que permitan su simulación por computadora, para con ello determinar las proyecciones que el modelo genera.

Para el desarrollo del Diagrama de Forrester corresponde clasificar las variables en flujos o niveles, según el Tabla 4. 
Las variables del diagrama causal del modelo planteado y las variables utilizadas para desarrollar el Diagrama de Forrester según Tabla 5.

Asimismo, con la finalidad de efectuar los cálculos de simulación se incluyeron las variables adicionales, ver Tabla 6.
La "operacionalización" de las variables expuestas, así como las ecuaciones y valores considerados para desarrollar los cálculos de simulación son expuestos en el Tabla 7 (referido a las variables del modelo causal) y el Tabla 8 (referido a las variables propuestas para efectos de simular el modelo).

Tabla 4. Tipos de variables en el Diagrama de Forrester

\begin{tabular}{ll}
\hline Tipo de variable & \multicolumn{1}{c}{ Definición } \\
\hline Niveles & $\begin{array}{l}\text { Variables acumulables (o desacumulables) en el tiempo, y que toman un valor en un momento } \\
\text { determinado. La acumulación (o desacumulación) se realiza a través de los flujos. }\end{array}$ \\
Flujos & $\begin{array}{l}\text { Los flujos pueden ser de entrada o de salida a un nivel, indican las velocidades de acumulación } \\
\text { (o desacumulación) en el transcurrir del tiempo. }\end{array}$ \\
Variable auxiliar & $\begin{array}{l}\text { Variables que permiten establecer nexos entre flujos y niveles que no guardan una relación } \\
\text { similar, en cuanto a las unidades que manejan. }\end{array}$ \\
\hline Fuente: [12]/Elaboración propia.
\end{tabular}

Tabla 5. Variables del Diagrama de Forrester, a partir del modelo causal

\begin{tabular}{|c|c|c|}
\hline Variable del modelo causal & Variable considerada en el Diagrama de Forrester & Tipo de variable \\
\hline Cambio climático & Prevención al CC & \\
\hline Desastres & Prevención a desastres & \\
\hline Eficacia del gasto en educación & Eficacia gasto & \\
\hline Eficacia del gasto en salud & Eficacia gasto & \\
\hline Eficiencia del gasto en educación & Eficiencia gasto & \\
\hline \multirow[t]{3}{*}{ Eficiencia del gasto en salud } & Eficiencia gasto & \\
\hline & Inversión en educación & \\
\hline & Tasa de no ingreso a AV x educación & \\
\hline & Personas que no entran a AV por influencia de la educación & \\
\hline & Porcentaje presupuesto MINEDU destinado a reducir brechas & \\
\hline Inversión en salud & Inversión en Salud & \\
\hline & Tasa Migración & \\
\hline \multicolumn{3}{|l|}{ Migración } \\
\hline Narco-terrorismo & Prevención a narcoterrorismo & \\
\hline & Tasa de crecimiento personas en AV & \\
\hline $\mathbf{N}^{\circ}$ de personas en áreas vulnerables & Personas a AV & \\
\hline & Nº de Personas ubicadas en Áreas Vulnerables & \\
\hline \multirow{2}{*}{ Reubicación de personas } & Porcentaje de reubicación & \\
\hline & Personas reubicadas & \\
\hline \multirow[b]{2}{*}{ Vulnerabilidad a la pobreza } & Tasa de no ingreso a AV por reducción de vulnerabilidad & \\
\hline & Personas que no entran a AV al reducirse vulnerabilidad & \\
\hline
\end{tabular}

Fuente: [5] / Elaboración propia. 
Tabla 6. Variables incluidas en el Diagrama de Forrrester con la finalidad de efectuar los cálculos del modelo

\begin{tabular}{lll}
\hline \multicolumn{1}{c}{ Variable incluida } & \multicolumn{1}{c}{ Definición } \\
\hline $\mathbf{N}^{\circ}$ Personas que no entran a AV & $\begin{array}{l}\text { Personas que debido a la educación y a la reducción de la vulnerabilidad } \\
\text { a la pobreza no llegan a ubicarse en las áreas vulnerables. }\end{array}$ \\
Población & Personas que viven en el territorio peruano. \\
Tasa promedio crecimiento poblacional & Tasa de crecimiento de la población \\
Crecimiento poblacional & Personas que cada año ingresan a conformar la población. \\
\hline
\end{tabular}

Fuente: [5] / Elaboración propia.

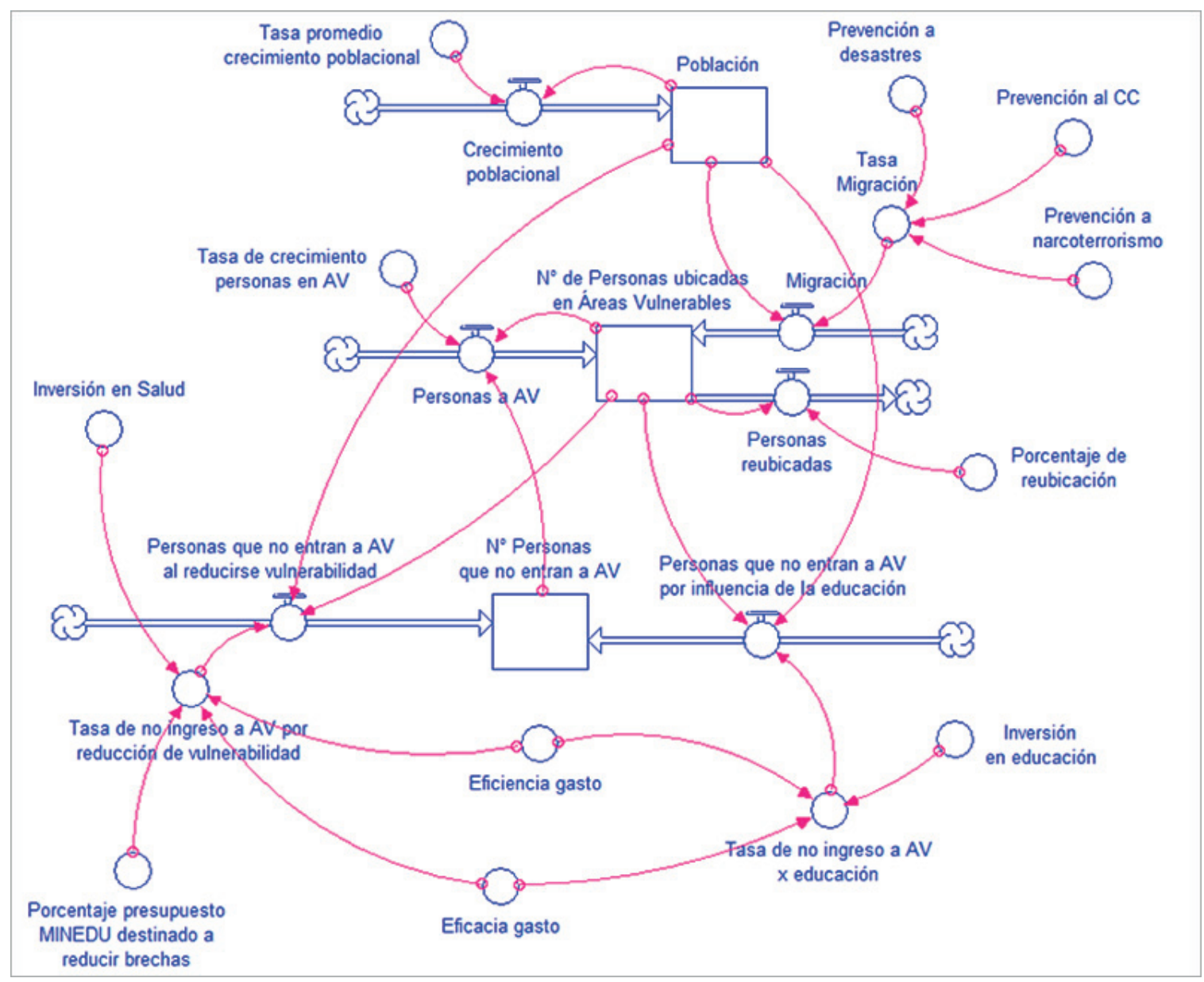

Figura 9. Diagrama de Forrester de las variables que impactarían el número de personas ubicadas en áreas vulnerables Elaboración propia.

El Diagrama de Forrester, considerando las variables expuestas es mostrado en la Figura 9.

Cabe anotar que el Diagrama de Forrester, así como las simulaciones presentadas en este trabajo se han realizado con el software Stella versión 10.1.2.

\subsection{Resultados de la simulación del modelo planteado}

El modelo propuesto señala que, de no tomarse ninguna acción, el número de personas ubicadas en áreas vulnerables ("N de personas en áreas vulnerables") aumentaría de manera acelerada desde 11’335,905 (11 millones 335 mil 905) personas en el año inicial (2016) hasta 33,967,813 (33 millones 967 mil 813), cincuenta años después.

La tendencia del número de personas en áreas vulnerables, indicada por el modelo de simulación, es mostrada en la Figura 10.

La figura mencionada señala que, de no tomarse ninguna acción, en 50 años el número de personas ubicadas en áreas vulnerables representaría el 51\% de la población total proyectada (la cual, en 50 años sería de 66291,189). 


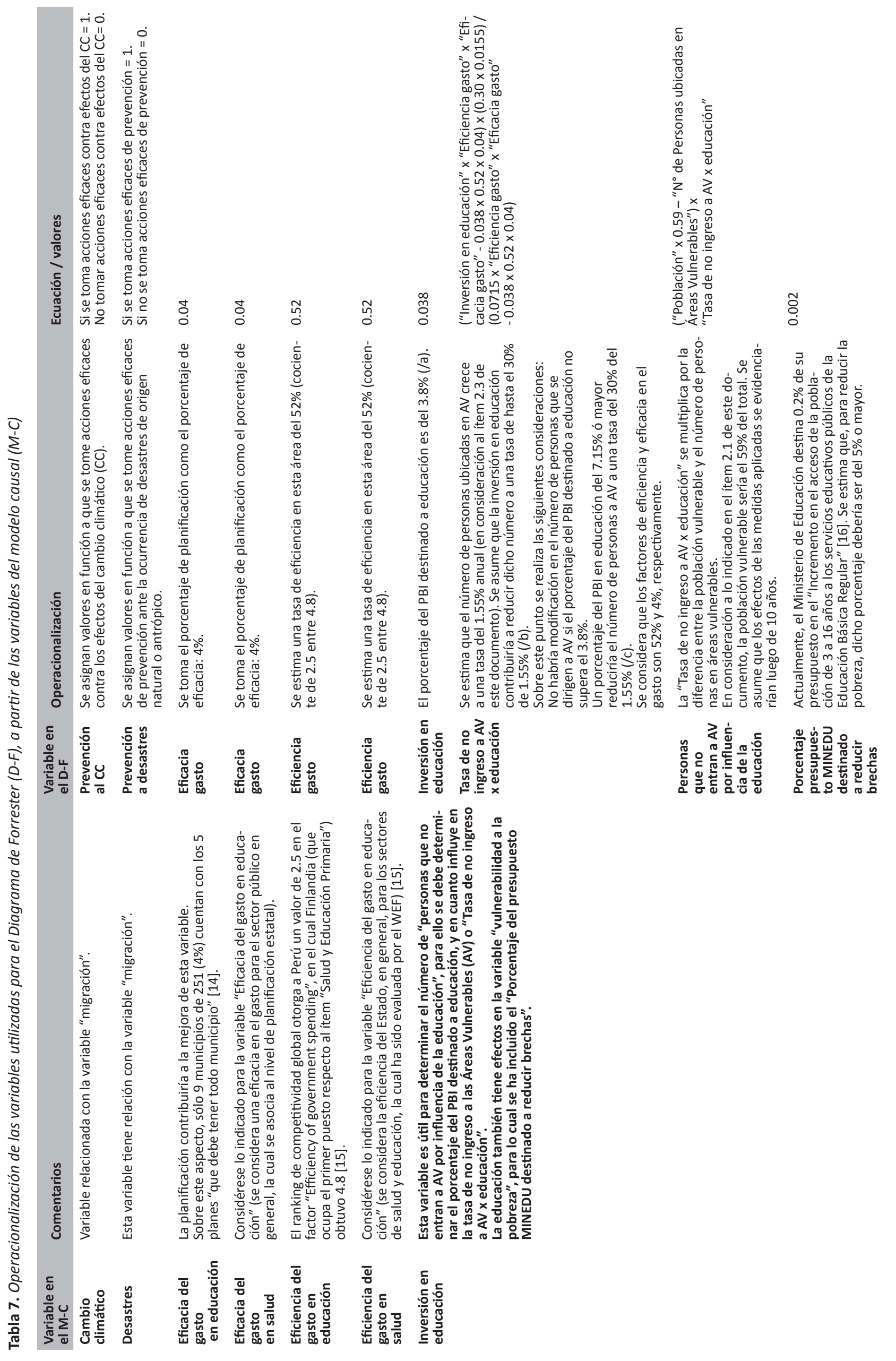




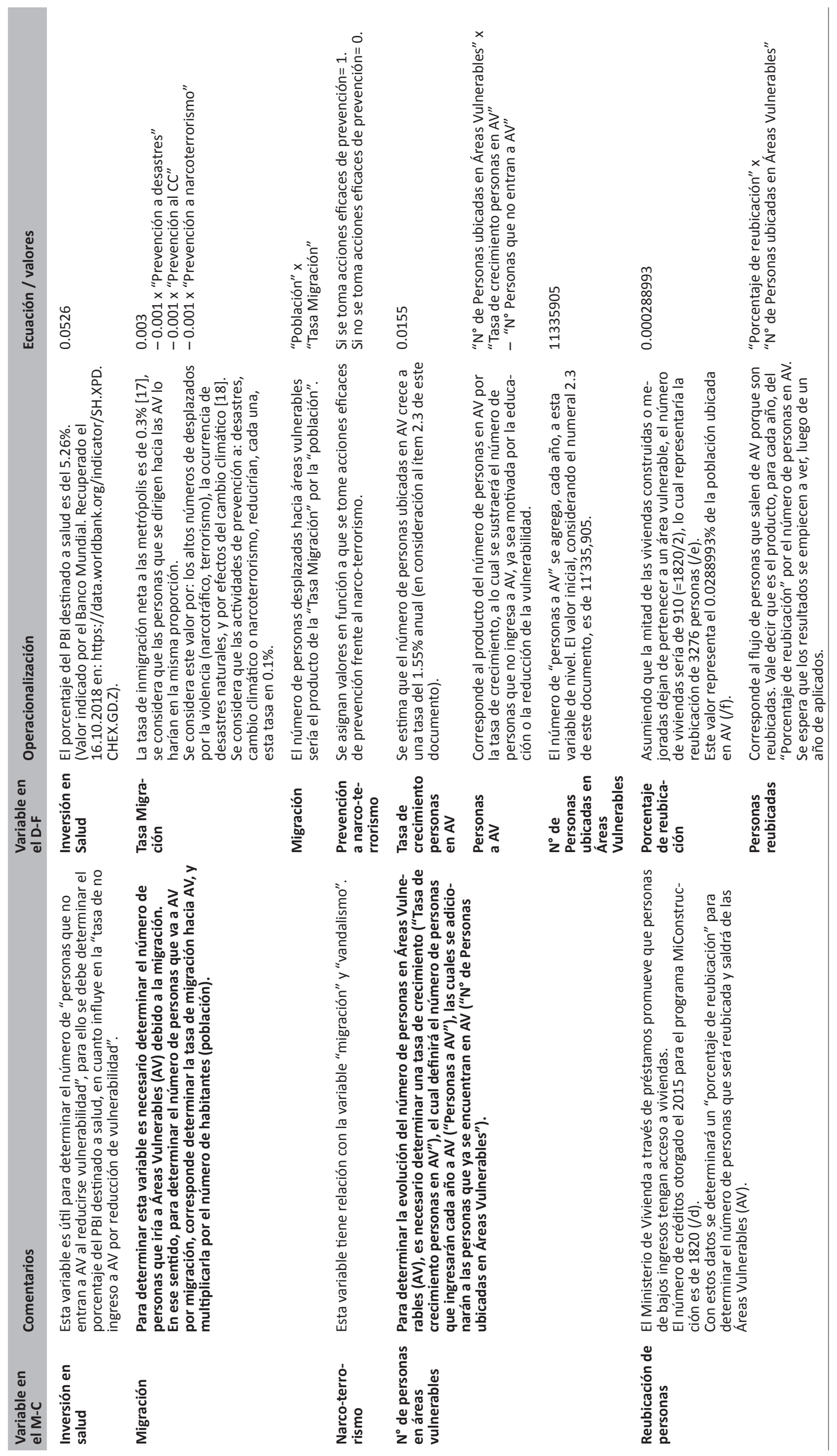




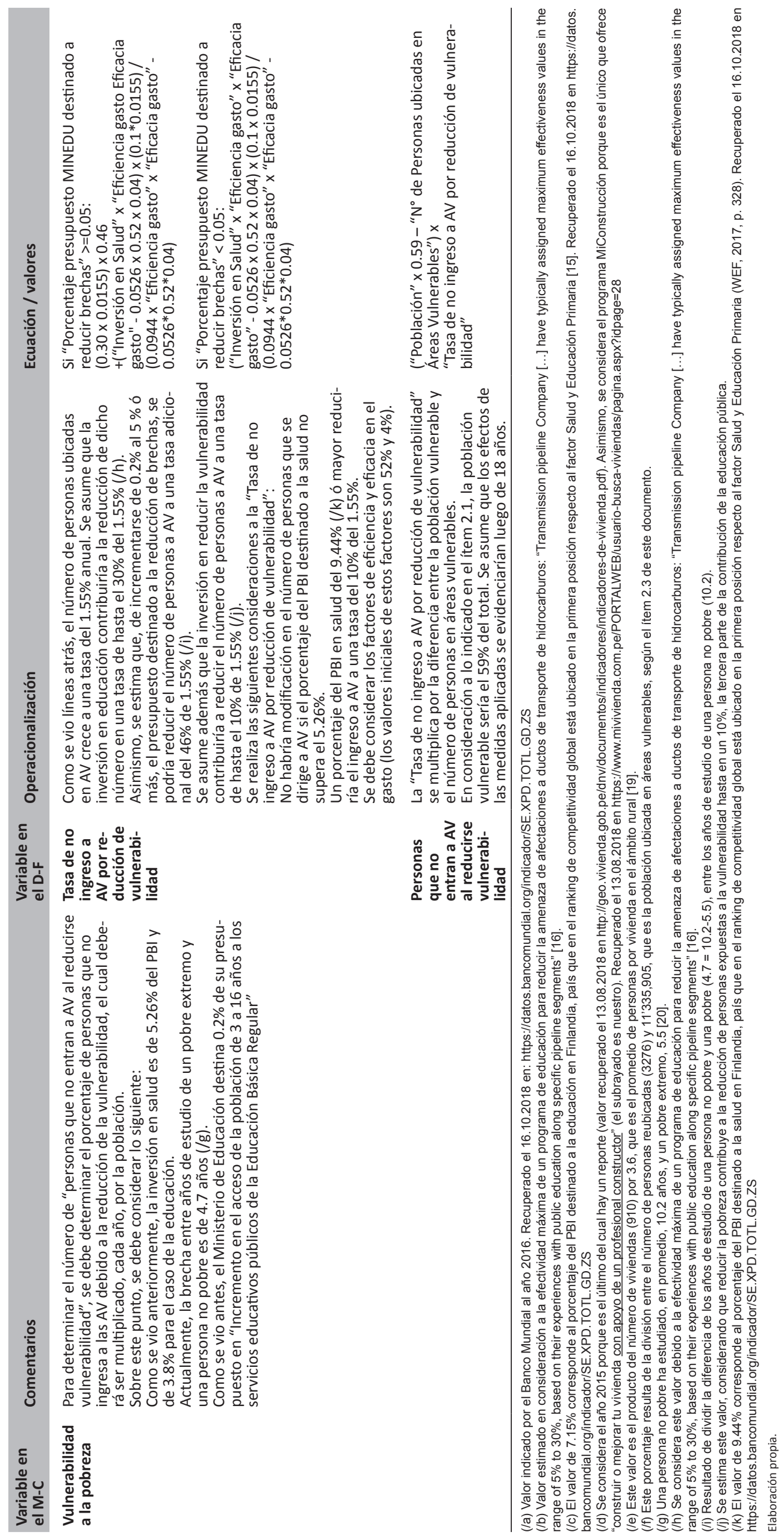


Tabla 8. Variables incluidas en el Diagrama de Forrrester (D-F) con la finalidad de efectuar los cálculos del modelo

\begin{tabular}{|c|c|c|c|c|}
\hline $\begin{array}{l}\text { Variable incluida } \\
\text { en el D-F }\end{array}$ & Definición & Comentarios & Operacionalización & $\begin{array}{l}\text { Ecuación / } \\
\text { valores }\end{array}$ \\
\hline $\begin{array}{l}\text { "N }{ }^{\circ} \text { Personas } \\
\text { que no entran } \\
\text { a AV" }\end{array}$ & $\begin{array}{l}\text { Número de personas que } \\
\text { debido a la educación y a } \\
\text { la reducción de la vulne- } \\
\text { rabilidad a la pobreza no } \\
\text { pasan a ubicarse en las } \\
\text { áreas vulnerables. }\end{array}$ & $\begin{array}{l}\text { Esta variable no está defi- } \\
\text { nida en el modelo causal, } \\
\text { pero es necesaria para el } \\
\text { Diagrama de Forrester, en } \\
\text { cuanto permite calcular las } \\
\text { variables del modelo. }\end{array}$ & $\begin{array}{l}\text { Esta variable de nivel recibe los flujos de las "per- } \\
\text { sonas que no entran a AV al reducirse vulnerabi- } \\
\text { lidad" y de las "personas que no entran a AV por } \\
\text { influencia de la educación" } \\
\text { Su valor inicial, para efectos de la simulación, es } \\
\text { cero. }\end{array}$ & 0 \\
\hline \multirow[t]{3}{*}{ “Población" } & \multirow[t]{3}{*}{$\begin{array}{l}\text { Personas que viven en el } \\
\text { territorio peruano. }\end{array}$} & \multirow{3}{*}{$\begin{array}{l}\text { Esta variable no está defi- } \\
\text { nida en el modelo causal, } \\
\text { pero es necesaria para el } \\
\text { Diagrama de Forrester, en } \\
\text { cuanto permite calcular las } \\
\text { variables del modelo. } \\
\text { Para determinar la pobla- } \\
\text { ción, se toma la tasa neta de } \\
\text { crecimiento poblacional, y } \\
\text { se determina el número de } \\
\text { personas que cada año se } \\
\text { agrega a la población. }\end{array}$} & $\begin{array}{l}\text { "Tasa promedio crecimiento poblacional” } \\
\text { La población crece a una tasa promedio del 1.5\% } \\
\text { anual }[21] .\end{array}$ & 0.015 \\
\hline & & & $\begin{array}{l}\text { “Crecimiento poblacional” } \\
\text { El número de personas que cada año ingresa } \\
\text { a conformar la población es el producto de la } \\
\text { "Tasa promedio crecimiento poblacional" por la } \\
\text { población. }\end{array}$ & $\begin{array}{l}\text { "Tasa promedio } \\
\text { crecimiento } \\
\text { poblacional" x } \\
\text { "Población" }\end{array}$ \\
\hline & & & $\begin{array}{l}\text { “Población” } \\
\text { El "crecimiento poblacional” se agrega, cada año, } \\
\text { a esta variable de nivel. El valor inicial de esta } \\
\text { variable es de } 31 \text { '488,625, que es la población } \\
\text { estimada para el año } 2016 \text { [6]. }\end{array}$ & 31488625 \\
\hline
\end{tabular}

Elaboración propia.

Tabla 9. Propuestas para reducir el número de personas en áreas vulnerables

\begin{tabular}{|c|c|c|}
\hline Escenario & Solución enfocada a... & Variables a mejorar \\
\hline Escenario 1 & Reducir el aumento del N de personas a AV por "Migración”. & $\begin{array}{l}\text { “Prevención a desastres". } \\
\text { "Prevención al CC". } \\
\text { "Prevención a narcoterrorismo". }\end{array}$ \\
\hline Escenario 2 & Aumentar el número de personas reubicadas de las AV. & "Porcentaje de reubicación". \\
\hline Escenario 3 & Promover la educación. & $\begin{array}{l}\text { "Inversión en educación”. } \\
\text { "Porcentaje presupuesto MINEDU destinado a reducir brechas". }\end{array}$ \\
\hline Escenario 4 & Reducir la vulnerabilidad a la pobreza. & $\begin{array}{l}\text { "Inversión en Salud". } \\
\text { "Porcentaje presupuesto MINEDU destinado a reducir brechas". }\end{array}$ \\
\hline Escenario 5 & Mejorar la eficacia en el gasto. & "Eficacia gasto". (*) \\
\hline Escenario 6 & Mejorar la eficiencia del gasto. & "Eficiencia gasto". $(* *)$ \\
\hline Escenario 7 & $\begin{array}{l}\text { Tratar el problema de la investigación de manera intensiva, en } \\
\text { varios aspectos. }\end{array}$ & $\begin{array}{l}\text { "Inversión en educación". } \\
\text { "Inversión en Salud". } \\
\text { "Porcentaje presupuesto MINEDU destinado a reducir brechas". } \\
\text { "Eficacia gasto". } \\
\text { "Eficiencia gasto". } \\
\text { "Porcentaje de reubicación". } \\
\text { "Prevención a desastres". } \\
\text { "Prevención al CC". } \\
\text { "Prevención a narcoterrorismo". }\end{array}$ \\
\hline
\end{tabular}

$\left(^{*}\right)$ Para efectos de este trabajo, se considera la eficacia en el gasto por parte del Estado para el sector público en general, la cual se asocia al nivel de planificación estatal (ver Apéndice 2).

$\left({ }^{* *}\right)$ Para efectos de este trabajo, se considera la eficiencia del Estado, en general, para los sectores de salud y educación, la cual ha sido evaluada por el World Economic Forum (ver Apéndice 2).

Fuente: [5] /

Elaboración propia.

\section{Propuestas para reducir el número de personas en áreas vulnerables}

En la tabla 9, se exponen diversos escenarios, para posteriormente indicar cuánto contribuiría cada uno de ellos en la reducción del número de personas ubicadas en áreas vulnerables.

Los valores utilizados para simular los escenarios planteados, así como las políticas propuestas a aplicar y su justificación, son expuestos en la Tabla 10.
Los resultados de las simulaciones son expuestos en las próximas líneas.

\subsection{Resultados de las propuestas de solución}

Los resultados de la simulación de los escenarios de solución planteados para la reducción del número de personas en áreas vulnerables (AV) son los siguientes:

5.1.1. Escenario 1: Solución enfocada a reducir el aumento del $\mathrm{N}^{\circ}$ de personas a AV por "Migración" 
Tabla 10. Valores considerados para la simulación de escenarios

\begin{tabular}{|c|c|c|c|c|}
\hline $\mathbf{N}^{\circ}$ & Variable a mejorar & Valor inicial & $\begin{array}{l}\text { Valor a } \\
\text { simular }\end{array}$ & Política / Acciones ( $*$ ) \\
\hline \multirow[t]{3}{*}{ Escenario 1} & Prevención a desastres. & 0 & 1 & Tomar acciones eficaces de prevención de desastres. \\
\hline & Prevención al CC. & 0 & 1 & Tomar acciones eficaces de prevención al cambio climático. \\
\hline & Prevención a narcoterrorismo. & 0 & 1 & Tomar acciones eficaces de prevención contra el narcoterrorismo \\
\hline Escenario 2 & Porcentaje de reubicación. & 0.000288993 & 0.014743066 & $\begin{array}{l}\text { Reubicar a las personas de las áreas vulnerables y/o coordinar para } \\
\text { que construyan de manera segura. } \\
\text { Considérese que el año } 2015 \text { se otorgó: } 98,221 \text { Bonos del programa } \\
\text { Techo Propio, } 35,594 \text { créditos del programa Mi Vivienda, y 1,820 } \\
\text { Créditos del programa Mi Construcción. } \\
\text { Acciones de política: } \\
\text { Orientar el total de créditos Mi Construcción (que es el único que } \\
\text { ofrece "construir o mejorar tu vivienda con apoyo de un profesional } \\
\text { constructor") a reubicar personas de las AV y/o a la construcción de } \\
\text { manera segura. } \\
\text { Orientar la tercera parte de los bonos Techo Propio y de los créditos } \\
\text { Mi Vivienda a la reubicación de personas en AV y/o a la construc- } \\
\text { ción de manera segura. } \\
\text { Lo anterior haría un total de } 46,424 \text { viviendas que salen de AV } \\
\text { (=98221/3+ 35594/3 + 1820), lo cual representaría la reubica- } \\
\text { ción de } 167,126(=46,424 \times 3.6 \text { ) personas. Este valor represen- } \\
\text { ta el 1.4743066\% de la población ubicada en AV (= } 167,126 / \\
\left.11^{\prime} 335,905\right) \text {. }\end{array}$ \\
\hline \multirow[t]{2}{*}{ Escenario 3} & Inversión en educación. & 0.038 & 0.07 & $\begin{array}{l}\text { Aumentar el presupuesto de la educación al } 7 \% \text { del PBI. } \\
\text { Integrar una visión abierta de la vulnerabilidad mediante la revalo- } \\
\text { rización de áreas geográficas, recuperación de recursos comunes y } \\
\text { del sentido de lugar e identidad. } \\
\text { Incidir en el reconocimiento de las consecuencias no percibidas. }\end{array}$ \\
\hline & $\begin{array}{l}\text { Porcentaje presupuesto MINEDU } \\
\text { destinado a reducir brechas }\end{array}$ & 0.002 & 0.05 & $\begin{array}{l}\text { Aumentar el presupuesto del MINEDU destinado a reducir brechas } \\
\text { a un } 5 \% \text {. }\end{array}$ \\
\hline \multirow[t]{2}{*}{ Escenario 4} & Inversión en Salud. & 0.0526 & 0.07 & Aumentar el presupuesto del sector salud al 7\% del PBI. \\
\hline & $\begin{array}{l}\text { Porcentaje presupuesto } \\
\text { MINEDU destinado a reducir } \\
\text { brechas. }\end{array}$ & 0.002 & 0.05 & $\begin{array}{l}\text { Aumentar el presupuesto del MINEDU destinado a reducir brechas } \\
\text { a un } 5 \% \text {. } \\
\text { Promover una perspectiva abierta, respecto a las vulnerabilidades. }\end{array}$ \\
\hline Escenario 5 & Eficacia gasto. & 0.04 & 0.20 & $\begin{array}{l}\text { Ejecutar proyectos de capacitación y de seguimiento destinados a } \\
\text { mejorar la eficacia del gasto. } \\
\text { El objetivo es mejorar la eficacia hasta un } 20 \% \text {. }\end{array}$ \\
\hline Escenario 6 & Eficiencia gasto. & 0.52 & 0.70 & $\begin{array}{l}\text { Ejecutar proyectos de capacitación y de seguimiento destinados a } \\
\text { mejorar la eficiencia del gasto. } \\
\text { El objetivo es mejorar la eficiencia hasta un } 70 \% \text {. }\end{array}$ \\
\hline \multirow[t]{9}{*}{ Escenario 7} & Inversión en educación. & 0.038 & 0.07 & Explicado para los escenarios anteriores. \\
\hline & Inversión en Salud. & 0.0526 & 0.07 & \\
\hline & $\begin{array}{l}\text { Porcentaje presupuesto MINEDU } \\
\text { destinado a reducir brechas. }\end{array}$ & 0.002 & 0.05 & \\
\hline & Eficacia gasto. & 0.04 & 0.20 & \\
\hline & Eficiencia gasto. & 0.52 & 0.70 & \\
\hline & Porcentaje de reubicación. & 0.000288993 & 0.014743066 & \\
\hline & Prevención a desastres. & 0 & 1 & \\
\hline & Prevención al CC. & 0 & 1 & \\
\hline & Prevención a narcoterrorismo & 0 & 1 & \\
\hline
\end{tabular}

Elaboración propia.

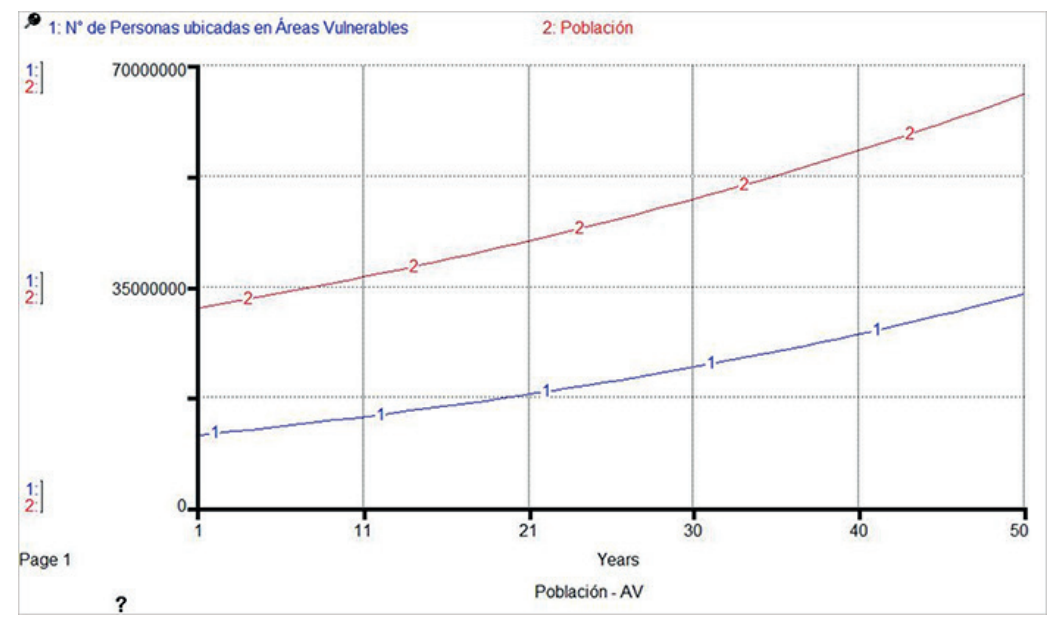

Figura 10. Escenario si no se toman acciones 


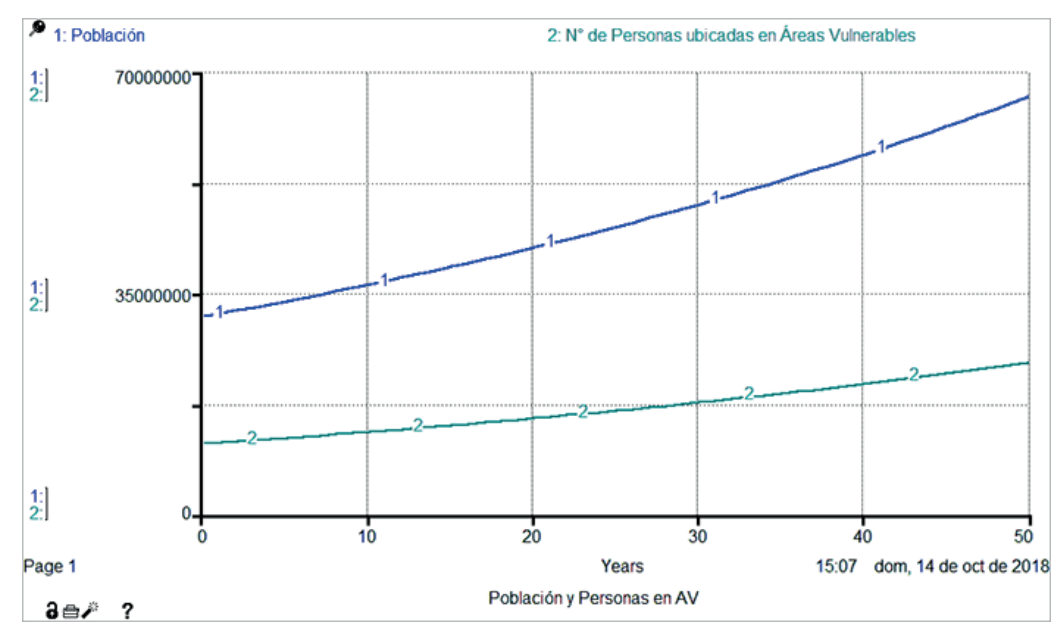

Figura 11. Escenario 1

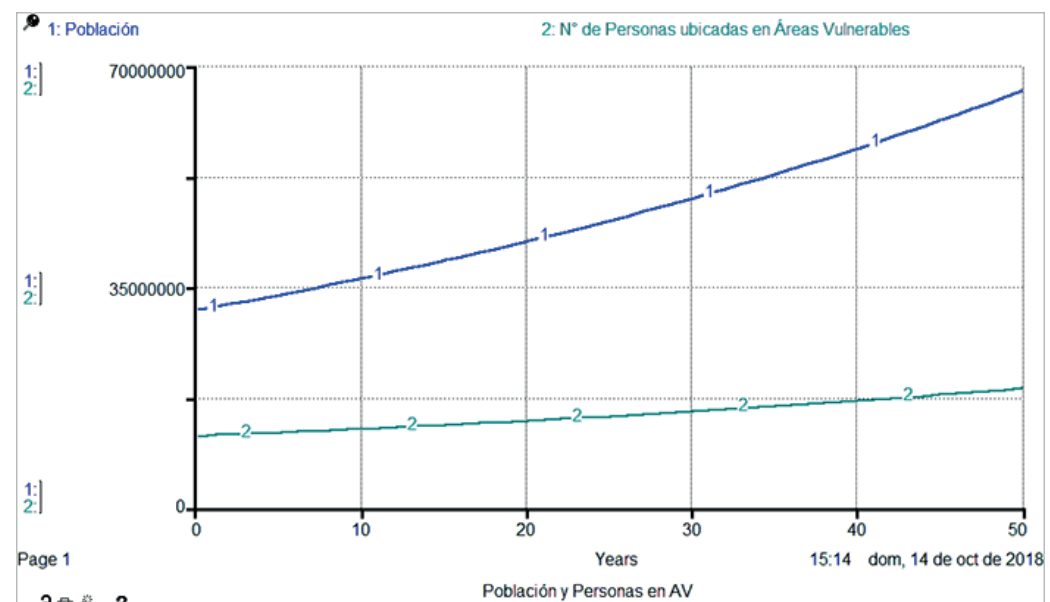

a星?

Figura 12. Escenario 2

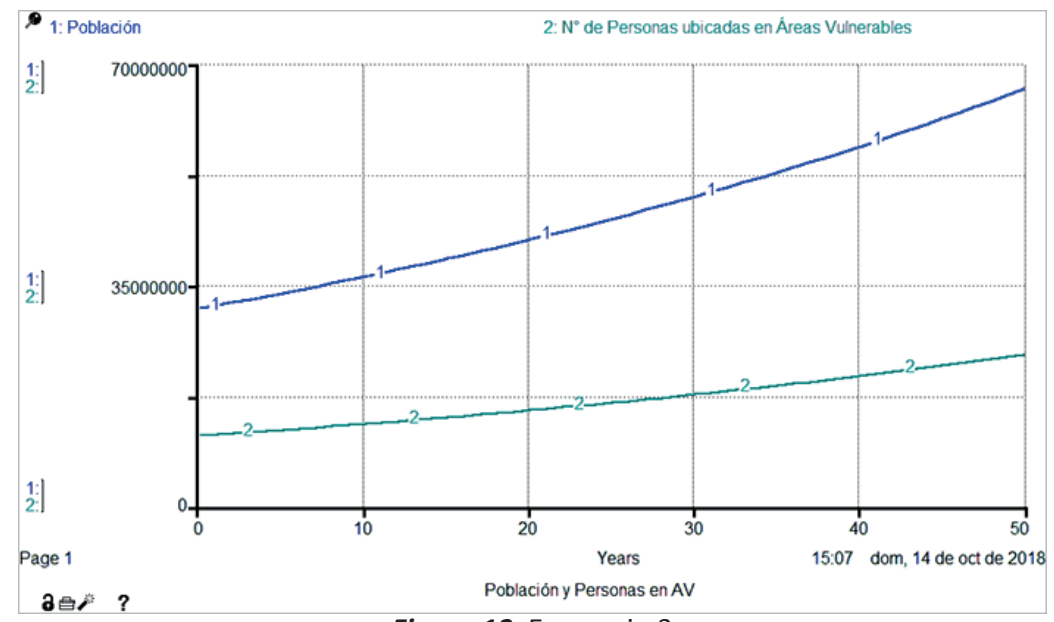

Figura 13. Escenario 3

Para este escenario, el " $\mathrm{N}^{\circ}$ de Personas ubicadas en Áreas Vulnerables” en 50 años sería de: 24’121,022. Lo cual corresponde al $36 \%$ de la población total proyectada (Ver Figura 11).

5.1.2. Escenario 2: Solución enfocada a aumentar el número de personas reubicadas de las AV

Para este escenario, el " $\mathrm{N}^{\circ}$ de Personas ubicadas en Áreas Vulnerables” en 50 años sería de: 19’021,119.
Lo cual corresponde al 29\% de la población total proyectada (Ver Figura 12).

5.1.3. Escenario 3: Solución enfocada a promover la educación

Para este escenario, el " $\mathrm{N}^{\circ}$ de Personas ubicadas en Áreas Vulnerables” en 50 años sería de: 21'248,351. Lo cual corresponde al $32 \%$ de la población total proyectada (Ver Figura 13). 


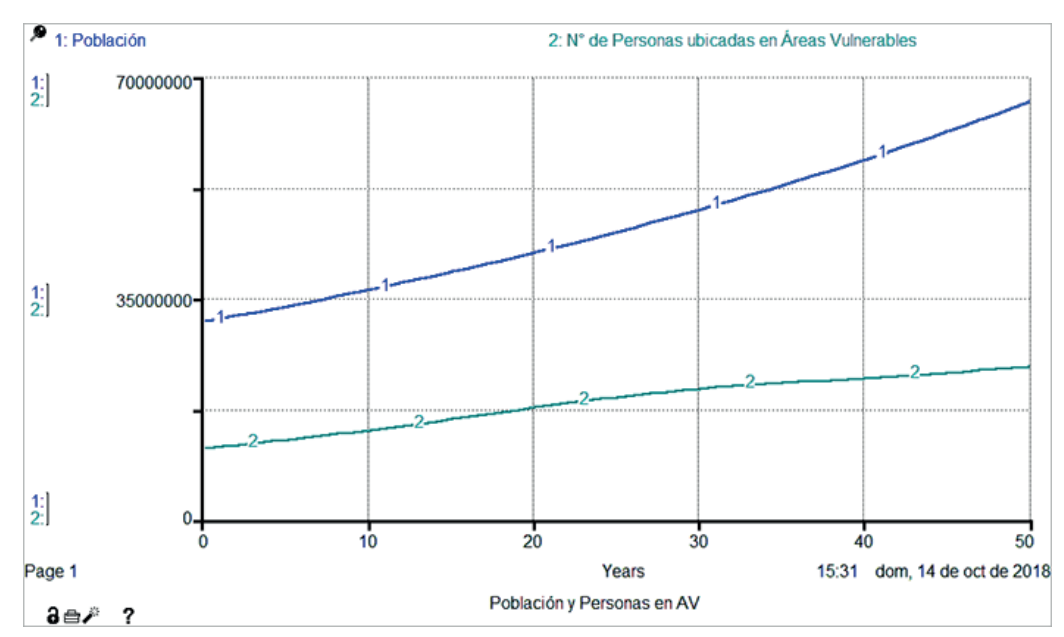

Figura 14. Escenario 4

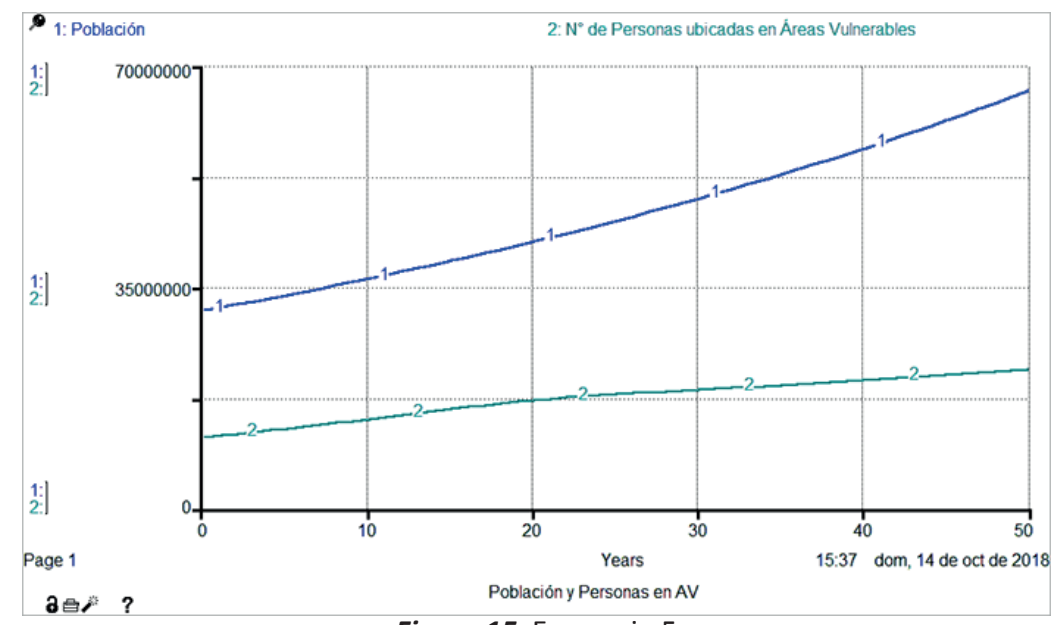

Figura 15. Escenario 5

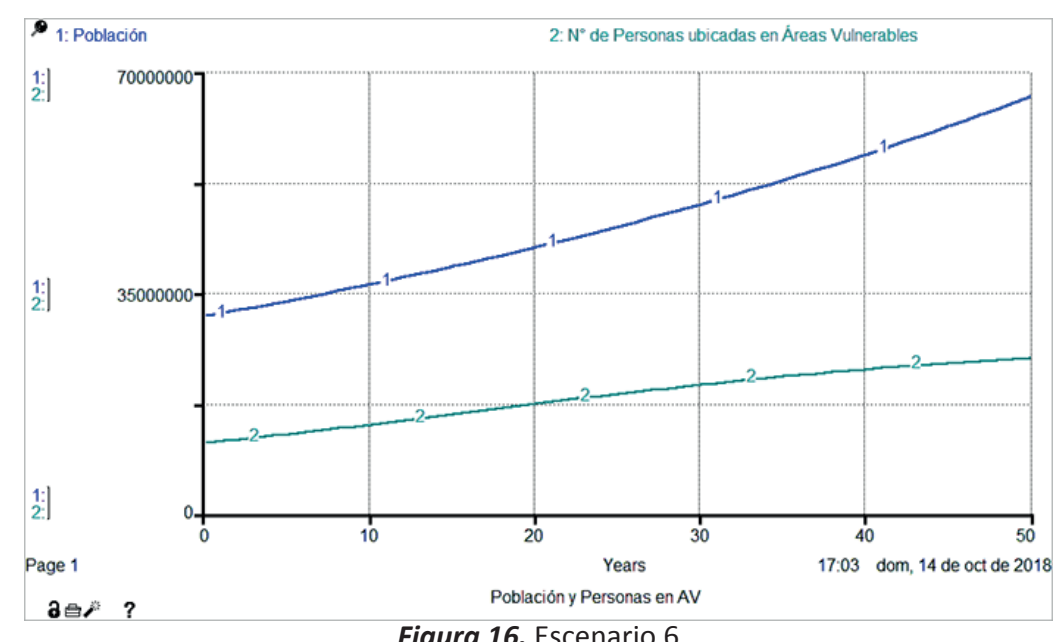

5.1.4. Escenario 4: Solución enfocada a reducir la vulnerabilidad a la pobreza

Para este escenario, el " $\mathrm{N}^{\circ}$ de Personas ubicadas en Áreas Vulnerables” en 50 años sería de: 24’260,895. Lo cual corresponde al $37 \%$ de la población total proyectada (Ver Figura 14).

5.1.5. Escenario 5: Solución enfocada a mejorar la eficacia en el gasto
Para este escenario, el "No de Personas ubicadas en Áreas Vulnerables” en 50 años sería de: 22’179,520. Lo cual corresponde al 33\% de la población total proyectada (Ver Figura 15).

5.1.6. Escenario 6: Solución enfocada a mejorar la eficiencia del gasto

Para este escenario, el " $\mathrm{N}^{\circ}$ de Personas ubicadas en Áreas Vulnerables” en 50 años sería de: 24’837,658. 


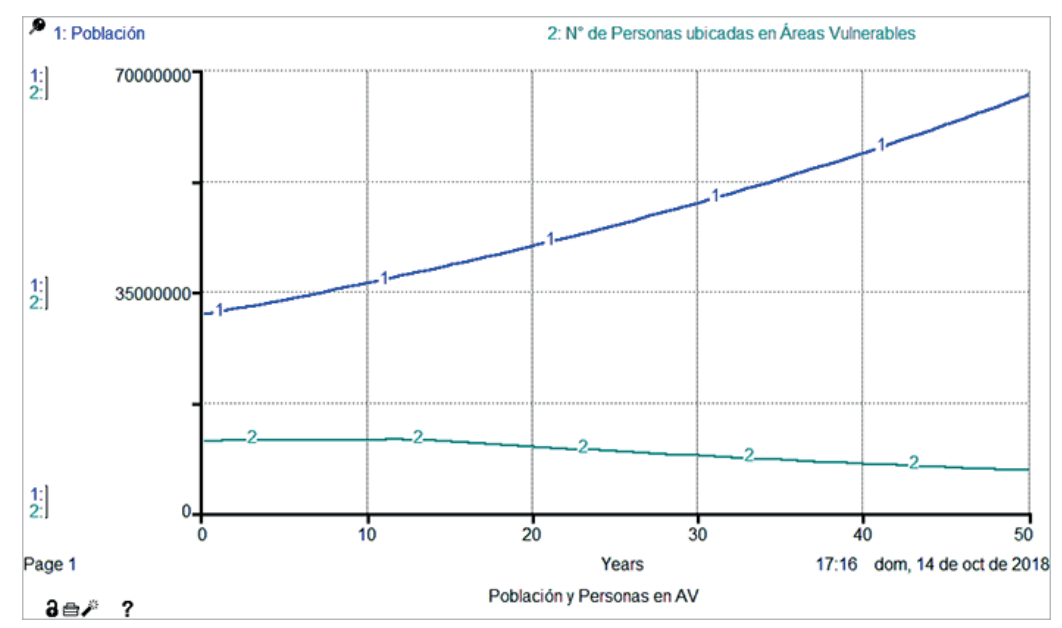

Figura 17. Escenario 7

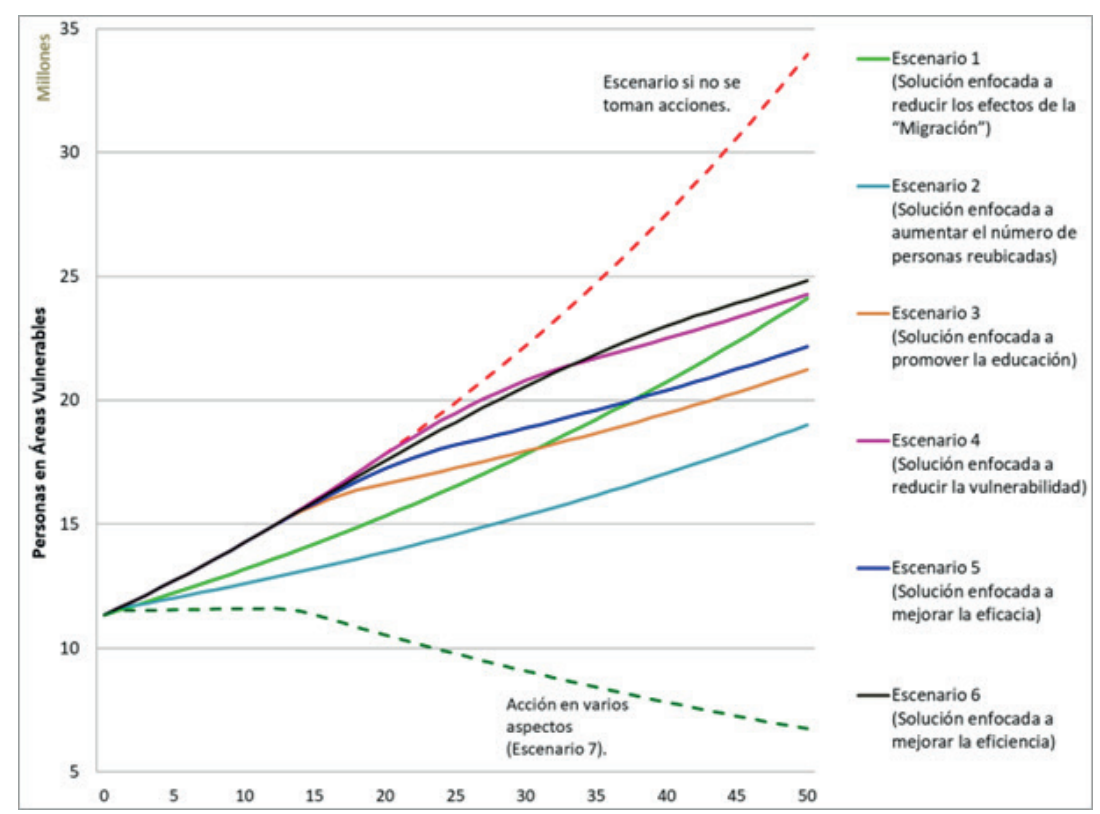

Figura 18. Número de personas en áreas vulnerables - comparación de escenarios

Lo cual corresponde al 37\% de la población total proyectada (Ver Figura 16).

5.1.7. Escenario 7: Solución enfocada a tratar el problema de la investigación de manera intensiva, en varios aspectos)

Para este escenario, el " $\mathrm{N}^{\circ}$ de Personas ubicadas en Áreas Vulnerables" en 50 años sería de: 67747,399. Lo cual corresponde al 10\% de la población total proyectada (Ver Figura 17).

\subsection{Comparación de escenarios}

En la Figura 18 se resume los resultados de los escenarios planteados:

De lo expuesto, la contribución de cada escenario en la reducción del número de personas en áreas vulnerables, es la siguiente [5]: $1^{\circ}$. Escenario 7: Solución enfocada a tratar el problema de la investigación de manera intensiva, en varios aspectos.

$2^{\circ}$. Escenario 2: Solución enfocada a aumentar el número de personas reubicadas de las áreas vulnerables.

$3^{\circ}$. Escenario 3: Solución enfocada a promover la educación.

4 . Escenario 5: Solución enfocada a mejorar la eficacia en el gasto.

$5^{\circ}$. Escenario 1: Solución enfocada a reducir el aumento del número de personas en áreas vulnerables por efectos de la migración (este escenario es el segundo que más contribuye a reducir el número de personas en áreas vulnerables hasta el año 30 ). 
Tabla 11. Variables de cada modelo de simulación, con la finalidad de reducir el número de personas en áreas vulnerables (AV)

\begin{tabular}{|c|c|c|c|c|c|c|c|c|}
\hline Variable & $\begin{array}{l}\text { Escenario si } \\
\text { no se toman } \\
\text { acciones }\end{array}$ & Escenario 1 & Escenario 2 & Escenario 3 & Escenario 4 & Escenario 5 & Escenario 6 & Escenario 7 \\
\hline Eficacia gasto & 0.04 & 0.04 & 0.04 & 0.04 & 0.04 & 0.2 & 0.04 & 0.2 \\
\hline Eficiencia gasto & 0.52 & 0.52 & 0.52 & 0.52 & 0.52 & 0.52 & 0.7 & 0.7 \\
\hline Inversión en educación & 0.038 & 0.038 & 0.038 & 0.07 & 0.038 & 0.038 & 0.038 & 0.07 \\
\hline Inversión en Salud & 0.0526 & 0.0526 & 0.0526 & 0.0526 & 0.07 & 0.0526 & 0.0526 & 0.07 \\
\hline Porcentaje de reubicación & 0.00028899 & 0.00028899 & 0.01474307 & 0.00028899 & 0.00028899 & 0.00028899 & 0.00028899 & 0.00028899 \\
\hline $\begin{array}{l}\text { Porcentaje presupuesto MINEDU } \\
\text { destinado a reducir brechas }\end{array}$ & 0.002 & 0.002 & 0.002 & 0.05 & 0.05 & 0.002 & 0.002 & 0.05 \\
\hline Prevención a desastres & 0 & 1 & 0 & 0 & 0 & 0 & 0 & 1 \\
\hline Prevención a narcoterrorismo & 0 & 1 & 0 & 0 & 0 & 0 & 0 & 1 \\
\hline Prevención al CC & 0 & 1 & 0 & 0 & 0 & 0 & 0 & 1 \\
\hline $\begin{array}{l}\text { Tasa de crecimiento personas } \\
\text { en AV }\end{array}$ & 0.0155 & 0.0155 & 0.0155 & 0.0155 & 0.0155 & 0.0155 & 0.0155 & 0.0155 \\
\hline $\begin{array}{l}\text { Tasa de no ingreso a AV por } \\
\text { reducción de vulnerabilidad }\end{array}$ & 0 & 0 & 0 & 0.002139 & 0.00278422 & 0.00077759 & 0.00037894 & 0.00325221 \\
\hline $\begin{array}{l}\text { Tasa de no ingreso a AV x } \\
\text { educación }\end{array}$ & 0 & 0 & 0 & 0.00444179 & 0 & 0.00221221 & 0.00105005 & 0.00454408 \\
\hline Tasa Migración & 0.003 & 0 & 0.003 & 0.003 & 0.003 & 0.003 & 0.003 & 0 \\
\hline $\begin{array}{l}\text { Tasa promedio crecimiento } \\
\text { poblacional }\end{array}$ & 0.015 & 0.015 & 0.015 & 0.015 & 0.015 & 0.015 & 0.015 & 0.015 \\
\hline
\end{tabular}

Elaboración propia.

Tabla 12. Número de personas en áreas vulnerables, por escenario

\begin{tabular}{|c|c|c|c|c|c|c|c|c|}
\hline Año & $\begin{array}{l}\text { Escenario si } \\
\text { no se toman } \\
\text { acciones }\end{array}$ & Escenario 1 & Escenario 2 & Escenario 3 & Escenario 4 & Escenario 5 & Escenario 6 & Escenario 7 \\
\hline 0 & $11,335,905$ & $11,335,905$ & $11,335,905$ & $11,335,905$ & $11,335,905$ & $11,335,905$ & $11,335,905$ & $11,335,905$ \\
\hline 1 & $11,606,077$ & $11,511,612$ & $11,606,077$ & $11,606,077$ & $11,606,077$ & $11,606,077$ & $11,606,077$ & $11,511,612$ \\
\hline 2 & $11,878,500$ & $11,686,715$ & $11,710,745$ & $11,878,500$ & $11,878,500$ & $11,878,500$ & $11,878,500$ & $11,520,325$ \\
\hline 3 & $12,156,505$ & $11,864,481$ & $11,816,931$ & $12,156,505$ & $12,156,505$ & $12,156,505$ & $12,156,505$ & $11,529,045$ \\
\hline 4 & $12,440,199$ & $12,044,952$ & $11,924,656$ & $12,440,199$ & $12,440,199$ & $12,440,199$ & $12,440,199$ & $11,537,772$ \\
\hline 5 & $12,729,690$ & $12,228,168$ & $12,033,945$ & $12,729,690$ & $12,729,690$ & $12,729,690$ & $12,729,690$ & $11,546,505$ \\
\hline 6 & $13,025,088$ & $12,414,171$ & $12,144,821$ & $13,025,088$ & $13,025,088$ & $13,025,088$ & $13,025,088$ & $11,555,245$ \\
\hline 7 & $13,326,505$ & $12,603,003$ & $12,257,306$ & $13,326,505$ & $13,326,505$ & $13,326,505$ & $13,326,505$ & $11,563,992$ \\
\hline 8 & $13,634,057$ & $12,794,707$ & $12,371,427$ & $13,634,057$ & $13,634,057$ & $13,634,057$ & $13,634,057$ & $11,572,745$ \\
\hline 9 & $13,947,860$ & $12,989,328$ & $12,487,206$ & $13,947,860$ & $13,947,860$ & $13,947,860$ & $13,947,860$ & $11,581,505$ \\
\hline 10 & $14,268,033$ & $13,186,908$ & $12,604,670$ & $14,268,033$ & $14,268,033$ & $14,268,033$ & $14,268,033$ & $11,590,271$ \\
\hline 11 & $14,594,695$ & $13,387,494$ & $12,723,842$ & $14,594,695$ & $14,594,695$ & $14,594,695$ & $14,594,695$ & $11,599,044$ \\
\hline 12 & $14,927,971$ & $13,591,132$ & $12,844,749$ & $14,927,971$ & $14,927,971$ & $14,927,971$ & $14,927,971$ & $11,607,824$ \\
\hline 13 & $15,267,986$ & $13,797,866$ & $12,967,417$ & $15,235,607$ & $15,267,986$ & $15,251,860$ & $15,260,331$ & $11,569,873$ \\
\hline 14 & $15,614,867$ & $14,007,746$ & $13,091,872$ & $15,517,260$ & $15,614,867$ & $15,566,254$ & $15,591,792$ & $11,483,705$ \\
\hline 15 & $15,968,743$ & $14,220,818$ & $13,218,140$ & $15,772,447$ & $15,968,743$ & $15,871,015$ & $15,922,364$ & $11,347,597$ \\
\hline 16 & $16,329,748$ & $14,437,131$ & $13,346,250$ & $16,000,539$ & $16,329,748$ & $16,165,969$ & $16,252,053$ & $11,180,298$ \\
\hline 17 & $16,698,016$ & $14,656,734$ & $13,476,228$ & $16,200,761$ & $16,698,016$ & $16,450,907$ & $16,580,858$ & $11,015,467$ \\
\hline 18 & $17,073,684$ & $14,879,678$ & $13,608,102$ & $16,372,179$ & $17,073,684$ & $16,725,586$ & $16,908,772$ & $10,853,065$ \\
\hline 19 & $17,456,891$ & $15,106,013$ & $13,741,902$ & $16,513,701$ & $17,456,891$ & $16,989,725$ & $17,235,783$ & $10,693,057$ \\
\hline 20 & $17,847,779$ & $15,335,790$ & $13,877,655$ & $16,634,281$ & $17,827,692$ & $17,237,124$ & $17,559,076$ & $10,535,409$ \\
\hline 21 & $18,246,494$ & $15,569,063$ & $14,015,391$ & $16,756,705$ & $18,185,981$ & $17,467,256$ & $17,878,569$ & $10,380,085$ \\
\hline 22 & $18,653,181$ & $15,805,884$ & $14,155,141$ & $16,881,003$ & $18,531,596$ & $17,679,521$ & $18,194,165$ & $10,227,050$ \\
\hline 23 & $19,067,993$ & $16,046,308$ & $14,296,932$ & $17,007,202$ & $18,864,327$ & $17,873,242$ & $18,505,754$ & $10,076,272$ \\
\hline 24 & $19,491,079$ & $16,290,388$ & $14,440,798$ & $17,135,331$ & $19,183,909$ & $18,047,661$ & $18,813,210$ & $9,927,717$ \\
\hline 25 & $19,922,598$ & $16,538,181$ & $14,586,768$ & $17,265,418$ & $19,490,019$ & $18,201,938$ & $19,116,394$ & $9,781,352$ \\
\hline 26 & $20,362,705$ & $16,789,744$ & $14,734,874$ & $17,397,493$ & $19,782,282$ & $18,335,143$ & $19,415,149$ & $9,637,145$ \\
\hline 27 & $20,811,563$ & $17,045,133$ & $14,885,148$ & $17,531,586$ & $20,060,258$ & $18,468,966$ & $19,709,300$ & $9,495,064$ \\
\hline 28 & $21,269,336$ & $17,304,406$ & $15,037,623$ & $17,667,727$ & $20,323,451$ & $18,604,836$ & $19,998,660$ & $9,355,078$ \\
\hline 29 & $21,736,189$ & $17,567,624$ & $15,192,331$ & $17,805,947$ & $20,571,298$ & $18,742,785$ & $20,283,019$ & $9,217,155$ \\
\hline 30 & $22,212,294$ & $17,834,845$ & $15,349,306$ & $17,946,277$ & $20,803,169$ & $18,882,844$ & $20,562,152$ & $9,081,266$ \\
\hline 31 & $22,697,824$ & $18,106,131$ & $15,508,582$ & $18,088,748$ & $21,018,368$ & $19,025,045$ & $20,835,813$ & $8,947,380$ \\
\hline 32 & $23,192,953$ & $18,381,543$ & $15,670,194$ & $18,233,393$ & $21,216,123$ & $19,169,419$ & $21,103,738$ & $8,815,468$ \\
\hline 33 & $23,697,862$ & $18,661,145$ & $15,834,176$ & $18,380,245$ & $21,395,590$ & $19,316,000$ & $21,365,642$ & $8,685,501$ \\
\hline 34 & $24,212,733$ & $18,945,000$ & $16,000,564$ & $18,529,336$ & $21,555,843$ & $19,464,820$ & $21,621,217$ & $8,557,451$ \\
\hline 35 & $24,737,751$ & $19,233,173$ & $16,169,394$ & $18,680,699$ & $21,706,332$ & $19,615,914$ & $21,870,134$ & $8,431,287$ \\
\hline 36 & $25,273,107$ & $19,525,729$ & $16,340,702$ & $18,834,370$ & $21,859,128$ & $19,769,314$ & $22,112,042$ & $8,306,984$ \\
\hline 37 & $25,818,991$ & $19,822,735$ & $16,514,526$ & $18,990,382$ & $22,014,266$ & $19,925,056$ & $22,346,564$ & $8,184,514$ \\
\hline 38 & $26,375,601$ & $20,124,258$ & $16,690,904$ & $19,148,771$ & $22,171,781$ & $20,083,175$ & $22,573,298$ & $8,063,849$ \\
\hline 39 & $26,943,136$ & $20,430,369$ & $16,869,873$ & $19,309,573$ & $22,331,709$ & $20,243,707$ & $22,791,817$ & 7,944,963 \\
\hline 40 & $27,521,799$ & $20,741,135$ & $17,051,473$ & $19,472,823$ & $22,494,086$ & $20,406,687$ & $23,001,666$ & $7,827,830$ \\
\hline 41 & $28,111,796$ & $21,056,629$ & $17,235,743$ & $19,638,558$ & $22,658,948$ & $20,572,152$ & $23,202,362$ & $7,712,424$ \\
\hline 42 & $28,713,338$ & $21,376,921$ & $17,422,722$ & $19,806,816$ & $22,826,333$ & $20,740,140$ & $23,393,391$ & $7,598,719$ \\
\hline 43 & $29,326,639$ & $21,702,086$ & $17,612,452$ & $19,977,634$ & $22,996,279$ & $20,910,689$ & $23,574,210$ & $7,486,691$ \\
\hline 44 & $29,951,917$ & $22,032,196$ & $17,804,974$ & $20,151,051$ & $23,168,823$ & $21,083,836$ & $23,746,588$ & $7,376,314$ \\
\hline 45 & $30,589,394$ & $22,367,328$ & $18,000,330$ & $20,327,106$ & $23,344,006$ & $21,259,621$ & $23,921,603$ & $7,267,565$ \\
\hline 46 & $31,239,296$ & $22,707,558$ & $18,198,561$ & $20,505,838$ & $23,521,866$ & $21,438,084$ & $24,099,297$ & $7,160,418$ \\
\hline 47 & $31,901,853$ & $23,052,962$ & $18,399,712$ & $20,687,288$ & $23,702,444$ & $21,619,264$ & $24,279,708$ & $7,054,852$ \\
\hline 48 & $32,577,298$ & $23,403,621$ & $18,603,825$ & $20,871,495$ & $23,885,780$ & $21,803,202$ & $24,462,877$ & $6,950,842$ \\
\hline 49 & $33,265,871$ & $23,759,614$ & $18,810,946$ & $21,058,503$ & $24,071,917$ & $21,989,940$ & $24,648,847$ & $6,848,365$ \\
\hline 50 & $33,967,813$ & $24,121,022$ & $19,021,119$ & $21,248,351$ & $24,260,895$ & $22,179,520$ & $24,837,658$ & $6,747,399$ \\
\hline
\end{tabular}

Elaboración propia. 
6․ Escenario 4: Solución enfocada a reducir la vulnerabilidad a la pobreza.

$7^{\circ}$. Escenario 6: Solución enfocada a mejorar la eficiencia en el gasto.

En la Tabla 11 se presentan las variables utilizadas para cada modelo; asimismo, en Tabla 12 se muestran los resultados del número de personas en las áreas vulnerables, para cada escenario

\section{Validación}

No es posible demostrar de manera rigurosa el modelo propuesto en este trabajo, como se indicó antes, el modelo simulado corresponde a una visión particular del mundo real. No obstante, se puede verificar que los resultados plantean situaciones coherentes respecto al número de personas en Áreas Vulnerables. De otra parte, como se verá más adelante, los resultados obtenidos al modificar las variables muestran consistencia lógica con los supuestos del modelo.

Cabe recalcar que el objetivo del modelo no es predecir resultados exactos, sino describir los comportamientos de ciertas variables en el futuro, dependiendo de las acciones que tomemos en el presente.[5]

\section{Discusión}

En este trabajo se propone un modelo para evaluar el aumento del número de personas en áreas vulnerables, describiéndose la situación problemática y evaluándose, mediante la simulación por computadora, los resultados de aplicar diferentes políticas públicas en el transcurrir del tiempo, con cierto rango de error esperado.

Es necesario considerar, sin embargo, que este trabajo presenta limitaciones como: la carencia de datos, o la falta de experiencia en la aplicación de modelos de simulación para analizar y evaluar políticas públicas.

Asimismo, no se puede soslayar que la simulación presenta algunas desventajas como: la imposibilidad de asegurar que un modelo sea válido (dado que es una representación del mundo real, puede no describirlo de manera adecuada), es riesgoso tomar medidas basadas en conclusiones provenientes de un modelo que no representa la realidad, la carencia de un criterio científico para seleccionar las alternativas a simular, la posibilidad de elaborar un andamiaje de pruebas y resultados falsos, basados en un modelo confiable, pero válido para otras condiciones [11].

En contraposición a lo indicado, un buen ejercicio de simulación nos permite: adquirir una experiencia rápida, a bajo costo y sin riesgos, del comportamiento de un sistema; identificar áreas con problemas en un sistema complejo; estudiar de manera sistemática las alternativas de solución propuestas, entre otras ventajas. Asimismo, se debe considerar que todo sistema, por complejo que sea, puede ser modelizado, y sobre ese modelo es posible ensayar alternativas [11].

De otra parte, la exposición de personas a situaciones de riesgo no es un problema que se pueda soslayar, puesto que involucra a un sector importante de la población (36\% del total según lo estimado por el MINAM). En ese sentido, este trabajo aporta en la atención de este problema, debido a que considera al número de personas en áreas vulnerables, como una variable de investigación. [5] De igual forma, este trabajo también aporta a la academia, al utilizar para el tratamiento de un problema de índole social y de evaluación de políticas públicas, una herramienta poco utilizada en estas áreas, como es la simulación por computadora.

Cabe manifestar que el tema abordado (la población expuesta a riesgos), es demasiado amplio para abordarlo de manera completa en un artículo de investigación, por lo cual, se espera que haya más trabajos y aportes en este sentido.

Adicionalmente, cabe indicar que ningún modelo causal, y éste no es la excepción, es capaz de predecir o de dar resultados exactos de la evolución de sus variables en el tiempo.

\section{Conclusiones}

- De no tomarse ninguna acción, el número de personas ubicadas en áreas vulnerables podría llegar a ser, en un plazo de cinco décadas, el 51\% de la población total.

- Según los resultados de esta investigación, las políticas que más aportarían a solucionar el problema del número de las personas ubicadas en áreas vulnerables, son las siguientes:

- Políticas orientadas a aumentar el número de personas reubicadas de las AV.

- Políticas orientadas a promover la educación.

- Políticas orientadas a mejorar la eficacia en el gasto.

No obstante lo indicado, debe considerarse que si vemos cada problema de manera aislada, y tratamos de atender cada problema de manera aislada, podremos obtener soluciones de corto plazo, oportunistas y temporales, sin abordar los problemas más profundos, aquellos que producen desequilibrio [13]. En ese sentido, la mejor alternativa sería actuar sobre todas las variables consideradas en el diagrama causal expuesto en la Figura 8.

- Los resultados de la política aplicada para atender el problema del número de personas ubicadas en áreas vulnerables no se verán en el corto plazo.

Este aspecto debe ser considerado al evaluarse las alternativas de solución, el hecho que no haya resultados en el corto plazo no significaría que no haya avances en la resolución del problema. 
- Finalmente, se debe señalar que el modelo desarrollado se limita a describir una situación problemática particular y a evaluar los resultados de aplicar diferentes políticas, describiendo su comportamiento en el tiempo, con cierto rango de error esperado. Cabe reiterar que ningún modelo de evaluación, y éste no es la excepción, es capaz de prever con exactitud la evolución de sus variables en el tiempo [5].

\section{Referencias bibliográficas}

[1] MINAM (2015). Guía para la elaboración de estudios de evaluación de riesgos a la salud y el ambiente (ERSA) en sitios contaminados. Lima, Perú: Ministerio del Ambiente.

[2] MEF (2013). Conceptos asociados a la gestión del riesgo en un contexto de cambio climático: aportes en apoyo de la inversión pública para el desarrollo sostenible. Lima: Ministerio de Economía y Finanzas.

[3] Osinergmin (2014). Supervisión y fiscalización de las actividades de electricidad e hidrocarburos. Recuperado el 06 de octubre de 2018, de http://www.osinergmin.gob.pe/newweb/ uploads/Publico/OficinaComunicaciones/EventosRealizados/ForoApurimac/2/Supervision_y_fiscalizacion_de_las_ actividades_de_electricidad_e_hidrocarburos.pdf

[4] Meadows, D. H.; Meadows, D. L., Randers, J., and Behrens, W. (1972). The limits to growth. A report for the Club of Rome's project on the predicament of mankind. New York, USA: Potomac Associates - Universe Books.

[5] Robles, J. (2019). Población en Áreas Vulnerables y Política de Gestión de Riesgos: El caso del transporte de gas natural por ductos. Tesis para obtener el grado de Maestría en Política Social no publicada: Universidad Nacional Mayor de San Marcos.

[6] PCM (2014). Plan Nacional de Gestión del Riesgo de Desastres 2014-2021, aprobado por Decreto Supremo $N^{\circ} 034$ 2014-PCM. Lima: Presidencia del Consejo de Ministros.

[7] MINAM (2014). Mapa de susceptibilidad física de zonas propensas a inundaciones y deslizamientos en la costa y sierra frente a la ocurrencia de eventos hidrometeorológicos externos. Recuperado el 15 de octubre de 2018, de http:// sinia.minam.gob.pe/mapas/mapa-susceptibilidad-fisica-zonas-propensas-inundaciones-deslizamientos

[8] MINAM (2016). El Perú y el cambio climático. Tercera Comunicación Nacional del Perú a la Convención Marco de las Naciones Unidas sobre el Cambio Climático. Lima: Ministerio del Ambiente.
[9] Aracil, J. (1995). Dinámica de Sistemas. Madrid: Isdefe.

[10] Rodriguez, R. (abril de 2016). Dirección Estratégico Empresarial mediante la Dinámica de Sistemas. Sesión 5: Metodología de Dinámica de Sistemas. Seminario virtual realizado por el Instituto Andino e Sistemas. Lima: Instituto Andino e Sistemas.

[11] Ballón, E. (2010). Modelo Dinámico del Sector Económico Informal - Aplicación de la metodología de los sistemas suaves dinámicos. Tesis para obtener el Grado de Maestro en Ciencias con mención en Ingeniería de Sistemas. Lima: Universidad Nacional de Ingeniería.

[12] Rodriguez, R. (abril de 2016). Dirección Estratégico Empresarial mediante la Dinámica de Sistemas. Sesión 3: DS: Fundamentos y elaboración de modelos. Seminario virtual realizado por el Instituto Andino e Sistemas. Lima: Instituto Andino e Sistemas.

[13] Sen, A. (2009). The idea of justice. London, Great Britain: Penguin Group.

[14] Espinoza, A. y Fort R. (2017). Inversión sin planificación: la calidad de la inversión pública en los barrios vulnerables de Lima. Lima: Grupo de Análisis para el Desarrollo.

[15] WEF (2017); The Global Competitiveness Report 20172018; Geneva, Switzerland: World Economic Forum

[16] MINEDU (2017). Plan Operativo Institucional 2018 del Ministerio de Educación, aprobado por Resolución Ministerial N 437-2017-MINEDU. Lima: Ministerio de Educación.

[17] Muhlbauer, K. (2014). Pipeline Risk Assessment: The Definitive Approach and Its Role in Risk Management. Pre-release version, 2014.

[18] INEI (2011). Perú: Migración Interna y el Sistema de Ciudades, 2002-2007. Lima: Instituto Nacional de Estadística e Informática.

[19] NRC (2018). Global report on internal displacement 2018; The Internal Displacement Monitoring Centre. Geneva, Switzerland: Norwegian Refugee Council.

[20] INEI (2015). Perú: Encuesta Demográfica y de Salud Familiar- ENDES, 2014. Lima: Instituto Nacional de Estadística e Informática.

[21] INEI (2017). Evolución de la Pobreza Monetaria, 2007-2016. Informe Técnico. Lima: Instituto Nacional de Estadística e Informática.

[22] INEI (2016). Perú: Síntesis Estadística 2016. Lima, Perú: Instituto Nacional de Estadística e Informática. 